\title{
Volume Comparison in the presence of a Gromov-Hausdorff $\varepsilon$-approximation II
}

\author{
Luca Sabatini ${ }^{1}$
}

\begin{abstract}
Let $(M, g)$ be any compact, connected, Riemannian manifold of dimension $n$. We use a transport of measures and the barycentre to construct a map from $(M, g)$ onto a Hyperbolic manifold $\left(\mathbb{H}^{n} / \Lambda, g_{0}\right)$ ( $\Lambda$ is a torsionless subgroup of $\left.\operatorname{Isom}\left(\mathbb{H}^{n}, g_{0}\right)\right)$, in such a way that its jacobian is sharply bounded from above. We make no assumptions on the topology of $(M, g)$ and on its curvature and geometry, but we only assume the existence of a measurable Gromov-Hausdorff $\varepsilon$-approximation between $\left(\mathbb{H}^{n} / \Lambda, g_{0}\right)$ and $(M, g)$. When the Hausdorff approximation is continuous with non vanishing degree, this leads to a sharp volume comparison, if $\varepsilon<\frac{1}{64 n^{2}} \min \left(i n j_{\left(\mathbb{H}^{n} / \Lambda, g_{0}\right)}, 1\right)$, then

$$
\begin{gathered}
\operatorname{Vol}\left(M^{n}, g\right) \geq \\
\left(1+160 n(n+1) \sqrt{\frac{\varepsilon}{\min \left(i n j_{\left(\mathbb{H}^{n} / \Lambda, g_{0}\right)}, 1\right)}}\right)^{\frac{n}{2}}|\operatorname{deg} h| \cdot \operatorname{Vol}\left(X^{n}, g_{0}\right) .
\end{gathered}
$$
\end{abstract}

AMS Subject Classification (2000). 53C23; 53C20; 58C35; 53B21

Keywords. Volumes comparison; Gromov-Hausdorff distance; energy bounds; barycentres

\footnotetext{
${ }^{1}$ Dipartimento di Scienze di Base ed Applicate per l'Ingegneria, Università degli Studi di Roma "La Sapienza", Italy luca.sabatini@sbai.uniroma1.it
} 


\section{Introduction}

As a corollary of the "Théorème Principal" (see. [1], p. 734), G. Besson, G. Courtois and S. Gallot proved the following

Theorem 1.1. (see. ibidem) Let $X$ and $Y$ be two connected compact orientable Riemannian manifolds of the same dimension $n$ and $f: Y \rightarrow X$ be a continuous map of non zero degree. If $X$ is endowed with some metric $g_{0}$ whose sectional curvature $K_{g_{0}}$ satisfies $K_{g_{0}} \leq-1$, then, for every metric $g$ on $Y$, one has ${ }^{2}$

(i)

$$
\operatorname{Ent}(Y, g) \operatorname{Vol}(Y, g) \geq|\operatorname{deg} f|(n-1)^{n} \operatorname{Vol}\left(X, g_{0}\right)
$$

(ii) If Ricci $(g) \geq-(n-1) g$, then $\operatorname{Vol}(Y, g) \geq|\operatorname{deg} f| \operatorname{Vol}\left(X, g_{0}\right)$

Moreover, in dimension $n \geq 3$ the equality is attained if and only if $(Y, g)$ and $\left(X, g_{0}\right)$ are the Real Hyperbolic Spaces and if there exists a locally isometric covering homotopic to $f$.

In this theorem, the property (ii) is an immediate consequence of the property (i) and of the Bishop's inequality.

In [6], (2006), G. Reviron proved the following

Theorem 1.2. Let $\left(M_{0}, g_{0}\right)$ be a compact Riemannian manifold of dimension $n$, whose systole $e^{3}$ is bounded from below by $\varepsilon_{0}$ and whose sectional curvature $K_{g_{0}}$ satisfies $K_{g_{0}} \leq-k^{2}$. Let $\left(Y, g_{*}\right)$ be a compact Riemannian manifold of dimension $n$ with strictly negative sectional curvature which is $\varepsilon_{0} / 13$-close to $\left(M, g_{0}\right)$ (with respect to the Gromov-Hausdorff distance) and whose systole is bounded from below by $\varepsilon_{0}$. Then for any other metric $g$ on $Y$ one has:

$$
\operatorname{Ent}(Y, g)^{n} \operatorname{Vol}(Y, g) \geq((n-1) k)^{n} \operatorname{Vol}\left(M_{0}, g_{0}\right) \text {. }
$$

In particular, when $(Y, g)$ is negatively curved, if $(Y, g)$ is $\left(\varepsilon_{0} / 13-\varepsilon^{\prime}\right)$-close to $\left(M, g_{0}\right)$ with $0<\varepsilon^{\prime}<\varepsilon_{0} / 13$, and if the systole of $(Y, g)$ is bounded from below by $\varepsilon_{0}$, then

$$
\operatorname{Vol}(Y, g) \geq\left(\frac{(n-1) k}{\operatorname{Ent}\left(M_{0}, g_{0}\right)}\right)^{n}\left(1-\frac{3 \varepsilon}{\varepsilon_{0}}\right)^{n} \operatorname{Vol}\left(M_{0}, g_{0}\right)
$$

\footnotetext{
${ }^{2}$ Recall that the Volumic Entropy is $\operatorname{Ent}(M, g)=\lim _{R \rightarrow+\infty} \frac{1}{R}\left[\operatorname{Vol}_{g} B_{M}\left(y_{0}, R\right)\right]$ being $B_{M}\left(y_{0}, R\right)$ the ball of radius $R$ centered in $y_{0} \in M$; it does not depend on the point $y_{0}$.

${ }^{3}$ The Systole is the length of the smallest closed geodesic not homotopic to zero.
} 
Vol. LVI (2018) Volumes Comparison in the presence of a Gromov-Hausdorff ...101

Proof. See [6], p. 30.

Notice that, with respect to the Theorem 1.1, in the Theorem 1.2 the existence of a map of non zero degree is not assumed, but deduced from the existence of a Gromov-Hausdorff approximation.

As in [7] (2013) and [8] (2016) any assumptions on the curvature of $(M, g)$ is done, we suppose only that one manifold has sectional curvature constant and equal to -1 (what we shall call a Hyperbolic Manifold) while on the second manifold no assumption on its curvature are done. As in the Euclidean case ([7]), we compare the volume of any compact connected Riemannian manifold $(M, g)$ with the one of a compact connected Hyperbolic Manifold $\left(X, g_{0}\right)$ of the same dimension, only assuming that the Gromov-Hausdorff distance between these two manifolds is smaller than some fixed positive $\varepsilon$; i.e. the estimate that we obtain is also valid for non small values of $\varepsilon$, but it is sharp when $\varepsilon$ goes to zero.

In the Hyperbolic space, if $\Lambda$ is a torsionless subgroup of $\operatorname{Isom}\left(\mathbb{H}^{n}\right.$, can $)$ which acts discretely and such that the quotient space $\left(X, g_{0}\right)=\left(\mathbb{H}^{n}\right.$, can $) / \Lambda$ is a manifold, three cases may occur:

1. the quotient manifold $\left(X, g_{0}\right)$ is compact;

2. the quotient manifold $\left(X, g_{0}\right)$ is noncompact with finite volume;

3. the quotient manifold $\left(X, g_{0}\right)$ is noncompact with infinite volume.

In the case 1 , the injectivity radius is always bounded from below by a strictly positive constant.

In the case 2 , the infimum, with respect $x \in X$, of the injectivity radius at the point $x$ is always equal to zero.

In the case 3 , there are manifolds whose injectivity radius is bounded from below, and other ones whose infimum of the injectivity radius is zero.

It is the reason why we shall only consider compact hyperbolic manifolds $\left(X, g_{0}\right)$ (case 1$)$, obtaining the main result of this paper:

Theorem 1.3. Let $\left(X, g_{0}\right)$ be a compact hyperbolic manifold and $(M, g)$ be any compact Riemannian manifold, if there exists a Gromov-Hausdorff $\varepsilon$-approximation $\left(X, g_{0}\right) \rightarrow(M, g)$ and if $\varepsilon<\frac{1}{64 n^{2}} \min \left[\operatorname{inj}\left(X, g_{0}\right), 1\right]$, then there exists a $C^{1}-$ map $H_{r}:(M, g) \rightarrow\left(X, g_{0}\right)$ satisfying the following properties:

(i) $H_{r}$ is a $\delta$-Hausdorff approximation, where $\delta=5 n \sqrt{\frac{\varepsilon}{\operatorname{inj}\left(X^{n}, g\right)}}+3 n \sqrt{\varepsilon}$. 
(ii) For every $y \in M$ and every orthonormal basis $\left\{\mathbf{e}_{i}^{\prime}\right\}_{1 \leq i \leq n}$ of $\left(T_{y} M, g_{y}\right)$,

$$
\sum_{i=1}^{n}\left\|d_{y} H_{r}\left(\mathbf{e}_{i}^{\prime}\right)\right\|^{2} \leq n\left(1+160 n(n+1) \sqrt{\frac{\varepsilon}{\min \left(\operatorname{inj}\left(X^{n}, g\right), 1\right)}}\right) .
$$

(iii) In every point, the Jacobian determinant of $H_{r}$ is bounded above by

$$
\left(1+160 n(n+1) \sqrt{\frac{\varepsilon}{\min \left(\operatorname{inj}\left(X^{n}, g\right), 1\right)}}\right)^{\frac{n}{2}} .
$$

Moreover, $H_{r}$ is explicitly constructed.

\section{The barycentre map for the Hyperbolic manifolds}

Let $\Lambda$ be a co-compact uniform lattice in the group of isometries of the Real Hyperbolic Space $\left(\mathbb{H}^{n}, \tilde{g}_{0}\right)$, where $\tilde{g}_{0}$ is the canonical metric of $\mathbb{H}^{n}$ whose sectional curvature is constant equal to -1 , then the quotient manifold $\left(X, g_{0}\right)=\left(\mathbb{H}^{n}, \tilde{g}_{0}\right) / \Lambda$ is a Hyperbolic Manifold with finite volume and injectivity radius $\varepsilon_{0}>0$. The space $\mathbb{H}^{n}$, with the canonical projection $p: \mathbb{H}^{n} \rightarrow X$ is the universal covering of the manifold $X$, with automorphismsgroup $G\left(\mathbb{H}^{n}, X\right) \cong \Lambda$. For the sake of simplicity, in the sequel we shall denote by an over-script ${ }^{\sim}$ all the elements of the universal covering $\mathbb{H}^{n}$, i.e. $\tilde{x} \in \mathbb{H}^{n}$.

Let $(X, d X)$ be a metric space and let $B(x, \alpha) \subset(X, d X)$ the ball centered at $x$ of radius $\alpha$; we consider the covering of $(X, d X)$ by a system of neighbourhoods $U_{\alpha}=B(x, \alpha)_{x \in X}$ of balls of fixed radius $\alpha$, that is denoted by $\left(X^{\alpha}, d X_{\alpha}\right)$ and let $p^{\alpha}:\left(X^{\alpha}, d X_{\alpha}\right) \rightarrow(X, d X)$ be the map of projection. Let $c \subset X$ be a closed loop based in $x_{0} \in X$; if $c$ can be lifted to $X^{\alpha}$ in a closed loop based in $x^{\alpha}=\left(p^{\alpha}\right)^{-1}\left(x_{0}\right)$, then

Definition 2.1. The $p^{\alpha}:\left(X^{\alpha}, d X_{\alpha}\right) \rightarrow(X, d X)$ is an $\alpha$-covering if and only if each closed loop $c$ of base point $x_{0} \in X$, is such that the class of $c$, denoted by $[c]$ coincides with the null class in $\pi_{1}\left(X, x_{0}\right) /\left(X, U_{\alpha}, x_{0}\right)$.

Let $(M, g)$ be a $n$-dimensional connected compact orientable Riemannian manifold without boundary and let $p^{\alpha}: M^{\alpha} \rightarrow M$ be a $\alpha$-covering of the manifold $M$. For the sake of simplicity, in what follows, we shall denote by $\bar{M}$ the manifold $M^{\alpha}$ and with an overline all the objects which live in this manifold: e.g. $\bar{y}$ is an element of $\bar{M}=M^{\alpha}$. 
Vol. LVI (2018) Volumes Comparison in the presence of a Gromov-Hausdorff ...103

We fix arbitrarily two real positive numbers $\alpha$ and $\varepsilon$ such that $0<5 \varepsilon<$ $\alpha<\frac{\varepsilon_{0}}{2}-\frac{3 \varepsilon}{2}$.

If the Gromov-Hausdorff distance between $X$ and $M$ is less than $\varepsilon$, the main result is the Theorem 1.3 whose proof is divided in several Lemmas occupying the most part of the present paper.

The first Lemma gives the relation between the distances on the bases when lifted to the covering spaces:

Lemma 2.1. ([5], theorem 3.33.) Let $\left(X^{n}, g_{0}\right)$ be a compact hyperbolic $n$-dimensional manifold, let $\varepsilon_{0}$ be its global injectivity radius and $\left(\mathbb{H}^{n}, \tilde{g}_{0}\right)$ be its Riemannian universal covering, let $\varepsilon<\frac{\varepsilon_{0}}{13}$ and $0<5 \varepsilon<\alpha<\frac{\varepsilon_{0}}{2}-\frac{3 \varepsilon}{2}$. Let $(M, g)$ be a n-dimensional connected compact orientable Riemannian manifold with $p^{\alpha}: \bar{M} \rightarrow M$ a $\alpha$-covering of the manifold $M$. If the GromovHausdorff distance between $X$ and $M$ is less than $\varepsilon$, then

1. there exists an isomorphism $\bar{r}$ between the group $G\left(\mathbb{H}^{n}, X\right) \cong \Lambda$ of automorphisms of the universal covering $p: \mathbb{H}^{n} \rightarrow X$ and the group $G(\bar{M}, M)$ of automorphisms of the $\alpha$-covering $p^{\alpha}: \bar{M} \longrightarrow M$;

2. there exist two maps:

$$
\begin{aligned}
& \tilde{f}: \mathbb{H}^{n} \longrightarrow \bar{M}, \\
& \tilde{h}: \bar{M} \longrightarrow \mathbb{H}^{n} ;
\end{aligned}
$$

which are lifts of the $\varepsilon$-approximations $f:\left(X, g_{0}\right) \rightarrow(M, g)$ and $h$ : $(M, g) \rightarrow\left(X, g_{0}\right)$ and which are equivariant with respect to the two actions of $\Lambda$ on $\mathbb{H}^{n}$ and on $\bar{M}$ (via the representation $\bar{r}$ ), i.e. $\forall \gamma \in$ $G\left(\mathbb{H}^{n}, X\right)$ and $\forall \zeta \in G(\bar{M}, M)$ :

$$
\begin{gathered}
\tilde{f} \circ \gamma=\bar{r}(\gamma) \circ \tilde{f}, \\
\tilde{h} \circ \zeta=(\bar{r})^{-1}(\zeta) \circ \tilde{h} ;
\end{gathered}
$$

moreover $\tilde{f}$ and $\tilde{h}$ are $\left(1+c_{1} \varepsilon, c_{2} \varepsilon\right)$-quasi isometries, more precisely they satisfy $\forall \bar{y} \in \bar{M}$ and $\forall \tilde{z} \in \mathbb{H}^{n}$ :

$$
\begin{gathered}
\varrho_{\mathbb{H}^{n}}(\tilde{h}(\bar{y}), \tilde{z}) \leq\left(1-\frac{3 \varepsilon}{\varepsilon_{0}}\right)^{-1} \varrho_{\bar{M}}(\bar{y}, \tilde{f}(\tilde{z}))+2 \varepsilon, \\
\varrho_{\bar{M}}(\bar{y}, \tilde{f}(\tilde{z})) \leq\left(1-\frac{3 \varepsilon}{2 \alpha}\right)^{-1} \varrho_{\mathbb{H}^{n}}(\tilde{h}(\bar{y}), \tilde{z})+2 \varepsilon,
\end{gathered}
$$




$$
\begin{array}{ll}
\forall \tilde{z} \in \mathbb{H}^{n}, \quad \varrho_{\mathbb{H}^{n}}((\tilde{h} \circ \tilde{f})(\tilde{z}), \tilde{z})<\varepsilon, \\
\forall \bar{y} \in \bar{M}, \quad \varrho_{\bar{M}}((\tilde{f} \circ \tilde{h})(\bar{y}), \bar{y})<\varepsilon .
\end{array}
$$

Proof. Apply Reviron [5], Theorem 3.33 (which is settled in a much more general context), noticing that

$$
\begin{gathered}
\left|\varrho_{\bar{M}}(\bar{y}, \tilde{f}(\tilde{z}))-\varrho_{\bar{M}}((\tilde{f} \circ \tilde{h})(\bar{y}), \tilde{f}(\tilde{z}))\right|<\varepsilon . \\
\left|\varrho_{\mathbb{H}^{n}}(\tilde{z}, \tilde{h}(\bar{y}))-\varrho_{\mathbb{H}^{n}}((\tilde{h} \circ \tilde{f})(\tilde{z}), \tilde{h}(\bar{y}))\right|<\varepsilon .
\end{gathered}
$$

To define the barycentre map it is necessary to construct an auxiliary function. Recall that the best choice to define the barycentre of a measure in a manifold whose sectional curvature is equal to -1 , is to chose the point $\tilde{x}$ where the function $\tilde{x} \mapsto \int_{\mathbb{H}^{n}} \cosh \varrho_{\mathbb{H}^{n}}(\tilde{x}, \tilde{z}) d \mu(\tilde{z})$ attains its minimum, we shall thus define the image of $\bar{y} \in \bar{M}$ by the so-called "barycentre map" as the unique point $\tilde{x}_{\bar{y}} \in \bar{M}$ where the function $\mathcal{B}_{\bar{y}}:\left(\mathbb{H}^{n}, \tilde{g}_{0}\right) \rightarrow \mathbb{R}^{+}$defined as:

$$
\mathcal{B}_{\bar{y}}(\tilde{x})=\int_{\mathbb{H}^{n}} \cosh \varrho_{\mathbb{H}^{n}}(\tilde{x}, \tilde{z}) e^{-c \varrho(\bar{y}, \tilde{f}(\tilde{z}))} d v_{\tilde{g}_{0}}(\tilde{z})
$$

attains its minimum.

Here $\bar{y}$ is a fixed point in $\bar{M}$, and $\varrho: \bar{M} \times \bar{M} \rightarrow \mathbb{R}^{+}$denotes an approximation of the distance function which satisfies the following properties:

1. $\varrho_{\bar{M}}-r \leq \varrho \leq \varrho_{\bar{M}}+r$, for a fixed $r>0$;

2. $\forall \gamma \in \operatorname{Isom}(\bar{M}, \bar{g})$, one has $\varrho(\gamma \bar{y}, \gamma \bar{z})=\varrho(\bar{y}, \bar{z})$;

3. $\|\nabla \varrho(\bar{y}, \bar{z})\| \leq 1+c_{1} r^{2}$, where the gradient is taken with respect to the first component $\bar{y}$.

Let $\mu$ be any measure on $\mathbb{H}^{n}$ such that

$$
\int_{\mathbb{H}^{n}} \cosh \left(\varrho_{\mathbb{H}^{n}}(\tilde{x}, \tilde{z})\right) d \mu(\tilde{z})>0
$$

and

$$
\int_{\mathbb{H}^{n}} e^{-\varrho_{\mathbb{H}^{n}(\tilde{x}, \tilde{z})} d \mu(\tilde{z})>0}
$$


Vol. LVI (2018) Volumes Comparison in the presence of a Gromov-Hausdorff ...105

for at least one $\tilde{x} \in \mathbb{H}^{n}$ (and thus, by the triangle inequality, for every $\left.\tilde{x} \in \mathbb{H}^{n}\right)$. Notice that this second condition is satisfied for any nonnegative non trivial measure $\mu$ on $\mathbb{H}^{n}$ such that there exists some $R \in \mathbb{R}^{+}$and $\tilde{x} \in \mathbb{H}^{n}$ such that $\mu\left(B_{\mathbb{H}^{n}}(\tilde{x}, R)\right)>0$, which means that the measure is not concentrate at infinity (i.e. it is a measure on $\mathbb{H}^{n}$ and not on its ideal boundary). Let us define the function $\mathcal{B}_{\mu}: \mathbb{H}^{n} \rightarrow \mathbb{R}^{+}$by

$$
\mathcal{B}_{\mu}(\tilde{x})=\int_{\mathbb{H}^{n}} \cosh \left(\varrho_{\mathbb{H}^{n}}(\tilde{x}, \tilde{z})\right) d \mu(\tilde{z})
$$

this function satisfies the

Lemma 2.2. $\quad$ 1. $\mathcal{B}_{\mu}$ is continuous (in fact $C^{2}$ ) and strictly convex.

2. Let $\tilde{\xi}$ be a fixed selected point of $\mathbb{H}^{n}$, then $\mathcal{B}_{\mu}(\tilde{x})$ goes to $+\infty$ when $\varrho_{\mathbb{H}^{n}}(\tilde{\xi}, \tilde{z}) \rightarrow \infty$.

3. $\mathcal{B}_{\mu}$ admits an absolute minimum and achieves this minimum at a unique point $\tilde{x}_{\mu}$, characterized by the equation:

$$
\int_{\mathbb{H}^{n}} \nabla\left(\cosh \varrho_{\mathbb{H}^{n}}\right)(\tilde{x}, \tilde{z}) d \mu(\tilde{z})=\mathbf{0},
$$

where the gradient is computed with respect to the first variable.

Proof of 1. Let $\mathbf{u}, \mathbf{v} \in T_{\tilde{x}} \mathbb{H}^{n}$ be two tangent vectors, it is sufficient to prove that $\mathcal{B}_{\mu}$ admits a (continuous) second derivative $\operatorname{Dd} \mathcal{B}_{\mu}(\mathbf{u}, \mathbf{v})$ which is positive definite. It is a classical result that, on the Hyperbolic Space, the Hessian of hyperbolic cosinus of the distance-function is continuous, because it satisfies

$$
\left.\operatorname{Dd}\left(\cosh \varrho_{\mathbb{H}^{n}}\right)\right|_{(\tilde{x}, \tilde{z})}(\mathbf{u}, \mathbf{v})=\cosh \left(\varrho_{\mathbb{H}^{n}}(\tilde{x}, \tilde{z})\right) \cdot \tilde{g}_{0}(\mathbf{u}, \mathbf{v})
$$

where the differential are taken, as usual, with respect to the first variable $\tilde{x}$. Let $\tilde{\xi}$ be any selected point of $\mathbb{H}^{n}$, for any point $\tilde{x} \in B_{\mathbb{H}^{n}}(\tilde{\xi}, 1)$, using $(2.5)$ and the triangle inequality, we can bound the first and the second derivatives of the integrand function from above by:

$$
\begin{gathered}
\left\|\nabla\left(\cosh \varrho_{\mathbb{H}^{n}}\right)(\tilde{x}, \tilde{z})\right\|=\sinh \left(\varrho_{\mathbb{H}^{n}}(\tilde{x}, \tilde{z})\right)\left\|\nabla \varrho_{\mathbb{H}^{n}}(\tilde{x}, \tilde{z})\right\| \leq \sinh \left(\varrho_{\mathbb{H}^{n}}(\tilde{x}, \tilde{z})+1\right) \\
\left|D d\left(\cosh \varrho_{\mathbb{H}^{n}}\right)_{(\tilde{x}, \tilde{z})}(\mathbf{v}, \mathbf{w})\right| \leq \cosh \left(\varrho_{\mathbb{H}^{n}}(\tilde{x}, \tilde{z})+1\right) \sqrt{\tilde{g}_{0}(\mathbf{v}, \mathbf{v})} \cdot \sqrt{\tilde{g}_{0}(\mathbf{w}, \mathbf{w})}
\end{gathered}
$$

where the right-hand sides are $\mu$-integrable functions which are independent on $\tilde{x}$; we may then apply the Lebesgue Dominate Derivation/Continuity theorems, which imply that $\mathcal{B}_{\mu}$ is $C^{2}$ and satisfies:

$$
\left.d \mathcal{B}_{\mu}\right|_{\tilde{x}}(\mathbf{v})=\int_{\mathbb{H}^{n}} \tilde{g}_{0}\left(\nabla\left(\cosh \varrho_{\mathbb{H}^{n}}\right)(\tilde{x}, \tilde{z}), \mathbf{v}\right) d \mu(\tilde{z}),
$$


and, by (2.5),

$$
\begin{gathered}
\left.D d \mathcal{B}_{\mu}\right|_{\tilde{x}}(\mathbf{v}, \mathbf{w})=\int_{\mathbb{H}^{n}} \operatorname{Dd}\left(\cosh \varrho_{\mathbb{H}^{n}}\right)_{(\tilde{x}, \tilde{z})}(\mathbf{v}, \mathbf{w}) d \mu(\tilde{z})= \\
\tilde{g}_{0}(\mathbf{v}, \mathbf{w}) \int_{\mathbb{H}^{n}} \cosh \left(\varrho_{\mathbb{H}^{n}}(\tilde{x}, \tilde{z})\right) d \mu(\tilde{z})=\tilde{g}_{0}(\mathbf{v}, \mathbf{w}) \mathcal{B}_{\mu} .
\end{gathered}
$$

It follows that $\left.\operatorname{Dd} \mathcal{B}_{\mu}\right|_{\tilde{x}}(\mathbf{v}, \mathbf{v})>0$ when $\mathbf{v} \neq 0_{\tilde{x}}$, and thus that $\mathcal{B}_{\mu}$ is strictly convex.

Proof of 2. Let $\tilde{\xi}$ be a fixed point of $\mathbb{H}^{n}$, applying the inequality $\cosh t \geq$ $\frac{1}{2} e^{t}$ and the triangle inequality, we get:

$$
2 \mathcal{B}_{\mu}(\tilde{x}) \geq \int_{\mathbb{H}^{n}} e^{\varrho_{\mathbb{H}^{n}}(\tilde{x}, \tilde{z})} d \mu(\tilde{z}) \geq \int_{\mathbb{H}^{n}} e^{\varrho_{\mathbb{H}^{n}}(\tilde{x}, \tilde{\xi})-\varrho_{\mathbb{H}} n(\tilde{\xi}, \tilde{z})} d \mu(\tilde{z}) \geq C \cdot e^{\varrho_{\mathbb{H}^{n}}(\tilde{x}, \tilde{\xi})}
$$

where $C=\int_{\mathbb{H}^{n}} e^{-\varrho_{\mathbb{H}^{n}}(\tilde{\xi}, \tilde{z})} d \mu(\tilde{z})$ is a strictly positive constant. We deduce that $\mathcal{B}_{\mu}(\tilde{x}) \rightarrow+\infty$ when $\varrho_{\mathbb{H} n}(\tilde{x}, \tilde{\xi}) \rightarrow+\infty$.

Proof of 3. As $\mathcal{B}_{\mu}$ is continuous and goes to $+\infty$ at infinity (by 1 . and 2.), it admits an absolute miminum and achieves this minimum; as $\mathcal{B}_{\mu}$ is strictly convex, this minimum is attained at a unique point, denoted by $\tilde{x}_{\mu}$; as $\mathcal{B}_{\mu}$ is strictly convex and $C^{1}$, this point is characterized by the equation $\nabla \mathcal{B}_{\mu}=\mathbf{0}$, which, by the formula (2.6), writes:

$$
\int_{\mathbb{H}^{n}} \nabla\left(\cosh \varrho_{\mathbb{H}^{n}}\right)\left(\tilde{x}_{\mu}, \tilde{z}\right) d \mu(\tilde{z})=\mathbf{0} .
$$

Let us now notice that the function $\mathcal{B}_{\bar{y}}$, defined at the beginning of this paper, coincides with $\mathcal{B}_{\mu}$ where $\mu=\mu_{\bar{y}}=e^{-c \varrho(\bar{y}, \tilde{f}(\bullet))} d v_{\tilde{g}_{0}}$. Let us now prove that $\mu_{\bar{y}}$ satisfies the two assumptions of the Lemma 2.2:

- it is obvious that $\int_{\mathbb{H}^{n}} e^{-\varrho_{\mathbb{H}^{n}}(\tilde{x}, \tilde{z})} d \mu_{\bar{y}}(\tilde{z})>0$, because the integral, to respect to $d v_{\tilde{g}_{0}}$, of a strictly positive continuous function is strictly positive.

- The second assumption is satisfied for every $c>\frac{n}{1-\frac{3 \varepsilon}{\varepsilon_{0}}}$ because of the

Lemma 2.3. The function $\mathcal{B}_{\bar{y}}$ is well defined for every $c>\frac{n}{1-\frac{3 \varepsilon}{\varepsilon_{0}}}$. 
Vol. LVI (2018) Volumes Comparison in the presence of a Gromov-Hausdorff ...107

Proof: By the property 1. of the approximation $\varrho$ of the distance function $\varrho_{\bar{M}}$ and by the the inequality (2.1) of the Lemma 2.1, we have:

$$
\begin{aligned}
0 & \leq \mathcal{B}_{\bar{y}}(\tilde{x}) \leq e^{c r} \int_{\mathbb{H}^{n}} e^{\varrho_{\mathbb{H}}(\tilde{x}, \tilde{z})} e^{-c \varrho_{\bar{M}}(\bar{y}, \tilde{f}(\tilde{z}))} d v_{\tilde{g}_{0}}(\tilde{z}) \leq \\
& \leq e^{c r} e^{2 c \varepsilon} \int_{\mathbb{H}^{n}} e^{\varrho_{\mathbb{H}^{n}}(\tilde{x}, \tilde{z})-c \varrho_{\mathbb{H}^{n}}(\tilde{h}(\bar{y}), \tilde{z})\left(1-\frac{3 \varepsilon}{\varepsilon_{0}}\right)} d v_{\tilde{g}_{0}}(\tilde{z}),
\end{aligned}
$$

and, by the triangle inequality, we obtain:

$$
\mathcal{B}_{\bar{y}} \leq e^{c\left(2 \varepsilon+r+\varrho_{\mathbb{H}^{n}}(\tilde{x}, \tilde{h}(\bar{y}))\right)} \int_{\mathbb{H}^{n}} e^{-\left(c\left(1-\frac{3 \varepsilon}{\varepsilon_{0}}\right)-1\right) \varrho_{\mathbb{H}^{n}}(\tilde{x}, \tilde{z})} d v_{\tilde{g}_{0}}(\tilde{z}) .
$$

We thus have only to prove that $\int_{\mathbb{H}^{n}} e^{-\beta \varrho_{\mathbb{H}^{n}}(\tilde{x}, \tilde{z})} d v_{\tilde{g}_{0}}(\tilde{z}) \leq+\infty$ if and only if $\beta>n-1$, which exactly means that the volume entropy of $\mathbb{H}^{n}$ is equal to $(n-1)$.

This proves that for every $c>\frac{n}{1-\frac{3 \varepsilon}{\varepsilon_{0}}}$, the measure $\mu_{\bar{y}}$ satisfies the assumptions of the Lemma 2.2; applying this Lemma, we get the

Lemma 2.4. For every $c>\frac{n}{1-\frac{3 \varepsilon}{\varepsilon_{0}}}$, the function $\mathcal{B}_{\bar{y}}$, defined by

$$
\int_{\mathbb{H}^{n}} \cosh \left(\varrho_{\mathbb{H}^{n}}(\tilde{x}, \tilde{z})\right) e^{-c \varrho(\bar{y}, \tilde{f}(\tilde{z}))} d v_{\tilde{g}_{0}}(\tilde{z})
$$

is a correctly defined (i.e. finite) $C^{2}$ function, which admits an absolute minimum and achieves this minimum at a unique point, denoted by $\tilde{x}_{\bar{y}}$, which is characterized by the equation

$$
\int_{\mathbb{H}^{n}} \nabla\left(\cosh \varrho_{\mathbb{H}^{n}}\right)(\tilde{x}, \tilde{z}) e^{-c \varrho(\bar{y}, \tilde{f}(\tilde{z}))} d v_{\tilde{g}_{0}}(\tilde{z})=\mathbf{0} .
$$

We define $\bar{H}: \bar{M} \rightarrow \mathbb{H}^{n}$ as the map which maps every $\bar{y} \in \bar{M}$ onto the point $\tilde{x}_{\bar{y}} \in \bar{M}$; this map will be called barycentre map because, if we consider the measure $\mu_{\bar{y}}=e^{-c \varrho(\bar{y}, \tilde{f}(\bullet))} d v_{\tilde{g}_{0}}(\bullet)$ on $\mathbb{H}^{n}$, then $\bar{H}(\bar{y})$ is the barycentre of $\mu_{\bar{y}}$ in the non-classical sense of [2].

The barycentre map is an equivariant almost-isometry, i.e. it is invariant under the actions of the elements of the isometry-group $\Lambda$ on $\mathbb{H}^{n}$ and, via its 
representation $\bar{r}$, on $\bar{M}$, thus it gives, by quotient, a map $H: M \rightarrow X$ which is a Hausdorff $A(n) \sqrt{\varepsilon}$-approximation if, as in the present case, the bases are compact $(A(n)$ is a constant depending only on the dimension $n)$. These two properties are synthesized in the following two Lemmas 2.5 and 2.9.

Lemma 2.5. The map $\bar{H}: \bar{M} \longrightarrow \mathbb{H}^{n}$ is equivariant with respect to the two actions of the group $\Lambda$ on $\mathbb{H}^{n}$ and on $\bar{M}$ via the representation $\bar{r}$, given by the Lemma 2.1; thus, making the quotient by the two actions of $\Lambda$ on $\mathbb{H}^{n}$ and on $\bar{M}$ it provides a map $H: M \rightarrow X$.

Proof. Let $G(\bar{M}, M)$ be the group of automorphisms of the $\alpha$-covering $p^{\alpha}: \bar{M} \rightarrow M$, from Lemma 2.1 it follows that there exists an isomorphic representation $\bar{r}: \Lambda \rightarrow G(\bar{M}, M)$ such that $\forall \gamma \in G\left(\mathbb{H}^{n}, X\right), \forall \tilde{x} \in \mathbb{H}^{n}$ we have $\tilde{f}(\gamma \tilde{x})=\bar{r}(\gamma) \tilde{f}(\tilde{x})$, we deduce that

$$
\begin{gathered}
\mathcal{B}_{\bar{r}(\gamma) \bar{y}}(\gamma \tilde{x})=\int_{\mathbb{H}^{n}} \cosh \varrho_{\mathbb{H}^{n}}(\gamma \tilde{x}, \tilde{z}) e^{-c \varrho(\bar{r}(\gamma) \bar{y}, \tilde{f}(\tilde{z}))} d v_{\tilde{g}_{0}}(\tilde{z}) \\
=\int_{\mathbb{H}^{n}} \cosh \varrho_{\mathbb{H}^{n}}\left(\tilde{x}, \gamma^{-1} \tilde{z}\right) e^{-c \varrho\left(\bar{y}, \bar{r}(\gamma)^{-1} \tilde{f}(\tilde{z})\right)} d v_{\tilde{g}_{0}}(\tilde{z}) \\
=\int_{\mathbb{H}^{n}} \cosh \varrho_{\mathbb{H}^{n}}\left(\tilde{x}, \gamma^{-1} \tilde{z}\right) e^{-c \varrho\left(\bar{y}, \tilde{f}\left(\gamma^{-1} \tilde{z}\right)\right)} d v_{\tilde{g}_{0}}(\tilde{z})=\mathcal{B}_{\bar{y}}(\tilde{x})
\end{gathered}
$$

because $d v_{\tilde{g}_{0}}(\tilde{z})$ is invariant by isometries. Then $\mathcal{B}_{\bar{r}(\gamma) \bar{y}}$ attains its minimum at $\gamma \tilde{x}_{\bar{y}}$ if and only if $\mathcal{B}_{\bar{y}}$ attains its minimum at $\tilde{x}_{\bar{y}}$. This implies that $\bar{H}(\bar{r}(\gamma) \bar{y})=\gamma \bar{H}(\bar{y})$, thus the map $\bar{H}$ is equivariant.

To prove that $\bar{H}$ is an almost-isometry, we shall prove a strongest property, i.e. that the point $\bar{H}(\bar{y})$ is close to $\tilde{h}(\bar{y})$; but we previously have to prove the following lemmas:

Lemma 2.6. If $\tilde{x}_{0}$ is a selected point of the Hyperbolic Space, then, for every $c>n$, the function

$$
\tilde{x} \mapsto \int_{\mathbb{H}^{n}} \cosh \varrho_{\mathbb{H}^{n}}(\tilde{x}, \tilde{z}) e^{-c \varrho_{\mathbb{H}^{n}}\left(\tilde{x}_{0}, \tilde{z}\right)} d v_{\tilde{g}_{0}}(\tilde{z}) .
$$

is correctly defined and achieves its minimum at $\tilde{x}=\tilde{x}_{0}$

Proof. Let $\nu=e^{-c \varrho_{\mathbb{H}} n\left(\tilde{x}_{0}, \bullet\right)} d v_{\tilde{g}_{0}}$, this measure satisfies the assumptions of the Lemma 2.2 if and only if $c>n$, In fact, as $\frac{1}{2} e^{t} \leq \cosh t \leq e^{t}$, $\int_{\mathbb{H}^{n}} \cosh \left(\varrho_{\mathbb{H}^{n}}\left(\tilde{x}_{0}, \tilde{z}\right)\right) d \nu(\tilde{z})$ is (up to a multiplicative constant) equivalent to $\int_{\mathbb{H}^{n}} e^{-(c-1) \varrho_{\mathbb{H}^{n}}\left(\tilde{x}_{0}, \tilde{z}\right)} d v_{\tilde{g}_{0}}(\tilde{z})$ which is finite if and only if $c-1>n-1$ (see the 
Vol. LVI (2018) Volumes Comparison in the presence of a Gromov-Hausdorff ...109

proof of the Lemma 2.3). Applying the Lemma 2.2 to the measure $\nu$, we obtain that, when $c>n$, the function

$$
\mathcal{B}_{\nu}: \tilde{x} \rightarrow \int_{\mathbb{H}^{n}} \cosh \left(\varrho_{\mathbb{H}^{n}}(\tilde{x}, \tilde{z})\right) e^{-c \varrho_{\mathbb{H}^{n}}\left(\tilde{x}_{0}, \tilde{z}\right)} d v_{\tilde{g}_{0}}(\tilde{z})
$$

is well defined, admits an absolute minimum and achieves this minimum at a unique point $\tilde{x}_{\nu}$, characterized by the equation

$$
\int_{\mathbb{H}^{n}} \nabla\left(\cosh \varrho_{\mathbb{H}^{n}}\right)\left(\tilde{x}_{\nu}, \tilde{z}\right) e^{-c \varrho_{\mathbb{H}^{n}}\left(\tilde{x}_{0}, \tilde{z}\right)} d v_{\tilde{g}_{0}}(\tilde{z})=\mathbf{0} .
$$

Writing everything with respect to the polar (exponential) coordinates centered at $\tilde{x}_{0}$ :

$$
\phi:\left\{\begin{array}{ccc}
] 0,+\infty\left[\times \mathbb{S}_{\tilde{x}_{0}}^{n-1}\right. & \rightarrow & \mathbb{H}^{n} \\
(r, \mathbf{u}) & \mapsto & \exp _{\tilde{x}_{0}}(r \cdot \mathbf{u})
\end{array}\right.
$$

(see the definitions in the proof of the Lemma 2.3), recalling that

$$
\varrho_{\mathbb{H}^{n}}(\tilde{x}, \phi(r, \mathbf{u}))=r, \quad\left(\nabla \varrho_{\mathbb{H}^{n}}\right)(\tilde{x}, \phi(r, \mathbf{u}))=-\mathbf{u}
$$

and

$$
\phi^{*} d v_{\tilde{g}_{0}}=(\sinh r)^{n-1} d r d v_{\mathbb{S}^{n-1}}
$$

we have:

$$
\begin{gathered}
\int_{\mathbb{H}^{n}} \nabla\left(\cosh \varrho_{\mathbb{H}^{n}}\right)\left(\tilde{x}_{0}, \tilde{z}\right) e^{-c \varrho_{\mathbb{H}^{n}}\left(\tilde{x}_{0}, \tilde{z}\right)} d v_{\tilde{g}_{0}}(\tilde{z})= \\
-\int_{0}^{+\infty}\left(\int_{\mathbb{S}_{\tilde{x}_{0}}^{n-1}} \mathbf{u} d v_{\mathbb{S}^{n-1}}(\mathbf{u})\right) e^{-c r} \sinh r(\sinh r)^{n-1} d r=\mathbf{0}
\end{gathered}
$$

because $\int_{\mathbb{S}_{\tilde{x}_{0}}^{n-1}} \mathbf{u} d v_{\mathbb{S}^{n-1}}(\mathbf{u})=\mathbf{0}$. This proves that $\tilde{x}_{0}$ satisfies the equation (2.8), and thus $\tilde{x}_{0}$ is the unique point where the function $\mathcal{B}_{\nu}$ attains its minimum, i.e. $\tilde{x}_{\nu}=\tilde{x}_{0}$.

Remark 2.1. The minimality of the map

$$
\tilde{x} \mapsto \int_{\mathbb{H}^{n}} \cosh \varrho_{\mathbb{H}^{n}}(\tilde{x}, \tilde{z}) e^{-c \varrho_{\mathbb{H}^{n}}\left(\tilde{x}_{0}, \tilde{z}\right)} d v_{\tilde{g}_{0}}(\tilde{z})
$$

expressed in the previous Lemma is nothing else than the fact that the barycentre map, seen as a map of the space $\mathbb{H}^{n}$ into itself is the identity-map of $\mathbb{H}^{n}$.

Before proving that the barycentre map is an almost-isometry it is necessary to prove two further sub lemmas: 
Lemma 2.7. Let $A B C$ be the geodesic triangle of the Real Hyperbolic Space whit edges of length $a, b$ and $c$, (as usual, the edge of length a is opposite to the vertex $A$ ); let $M$ be a point of the edge $B C$, then the following relation holds:

$$
\begin{gathered}
\cosh \varrho_{\mathbb{H}^{n}}(A, M)= \\
=\cosh \varrho_{\mathbb{H}^{n}}(A, B) \frac{\sinh \varrho_{\mathbb{H}^{n}}(M, C)}{\sinh \varrho_{\mathbb{H}^{n}}(B, C)}+\cosh \varrho_{\mathbb{H}^{n}}(A, C) \frac{\sinh \varrho_{\mathbb{H}^{n}}(M, B)}{\sinh \varrho_{\mathbb{H}^{n}}(B, C)} .
\end{gathered}
$$

Proof. Let $\bar{c}:[0, a] \rightarrow \mathbb{H}^{n}$, where $a=\varrho_{\mathbb{H}^{n}}(B, C)$ be the normal geodesic such that $\bar{c}(0)=B$ and $\bar{c}(a)=C$. We set $M(t)=\bar{c}(t)$ and $y(t)=$ $\cosh \varrho_{\mathbb{H}^{n}}(A, \bar{c}(t))$. The formula 2.6 implies

$$
y^{\prime \prime}(t)=\left.D d\left(\cosh \varrho_{\mathbb{H}^{n}}\right)\right|_{(\bar{c}(t), A)}\left(\bar{c}^{\prime}(t), \bar{c}^{\prime}(t)\right)=\cosh \left[\varrho_{\mathbb{H}^{n}}(\bar{c}(t), A)\right],
$$

thus $y(t)$ is a solution of the following boundary problem:

$$
\left\{\begin{array}{l}
y^{\prime \prime}(t)=y(t) \\
y(0)=\cosh \varrho_{\mathbb{H}^{n}}(A, B) \\
y(a)=\cosh \varrho_{\mathbb{H}^{n}}(A, C) .
\end{array}\right.
$$

It is clear that the function

$$
x(t)=\cosh \varrho_{\mathbb{H}^{n}}(A, B) \frac{\sinh (a-t)}{\sinh \varrho_{\mathbb{H}^{n}}(B, C)}+\cosh \varrho_{\mathbb{H}^{n}}(A, C) \frac{\sinh t}{\sinh \varrho_{\mathbb{H}^{n}}(B, C)}
$$

is the solution of the problem (2.10). The identification $y(t)=x(t)$ proves the relation (2.9).

Let $\mu^{\prime}(\bullet)$ be any finite measure without atoms on $\mathbb{H}^{n}$ satisfying the integrability condition: there exists some $\tilde{x}_{0} \in \mathbb{H}^{n}$ such that

$$
\int_{\mathbb{H}^{n}} e^{\varrho_{\mathbb{H}^{n}\left(\tilde{x}_{0}, \tilde{z}\right)} d \mu^{\prime}(\tilde{z})<+\infty}
$$

(notice that this condition implies that

$$
\int_{\mathbb{H}^{n}} \cosh \left(\varrho_{\mathbb{H}^{n}}\left(\tilde{x}_{0}, \tilde{z}\right)\right) d \mu^{\prime}(\tilde{z})<+\infty
$$

for every $\tilde{x} \in \mathbb{H}^{n}$ ), let $b$ be its barycentre, i.e. the point where the function $x \mapsto \int_{\mathbb{H}^{n}} \cosh \left(\varrho_{\mathbb{H}^{n}}(x, z)\right) d \mu^{\prime}(z)$ achieves its minimum and let us consider the geodesic triangle $z b x$, corresponding to the triangle of the previous lemma setting the identifications $A \equiv z, B \equiv b$ and $C \equiv x$. Let us denote by $c$ the normal geodesic $[0, a] \rightarrow \mathbb{H}^{n}$ joining the barycentre $b$ to the point $x$ and 
Vol. LVI (2018) Volumes Comparison in the presence of a Gromov-Hausdorff ...111

let $M$ be the point of the edge $b x$ at distance equal to $\eta$ from $b$. From the relation (2.9) we draw the equality:

$$
\begin{gathered}
\cosh \varrho_{\mathbb{H}^{n}}(x, z)-\cosh \varrho_{\mathbb{H}^{n}}(b, z)\left(\cosh a-\frac{\cosh \eta-1}{\sinh \eta} \sinh a\right) \\
=\frac{\sinh a}{\sinh \eta}\left[\cosh \varrho_{\mathbb{H}^{n}}(c(\eta), z)-\cosh \varrho_{\mathbb{H}^{n}}(b, z)\right] .
\end{gathered}
$$

As a consequence, we have:

Lemma 2.8. For any measure $\mu^{\prime}$ satisfying the above integrability conditions, let $b$ the barycentre of the measure $\mu^{\prime}$ and $x$ be any point of of $\mathbb{H}^{n}$, then

$$
\int_{\mathbb{H}^{n}} \cosh \left(\varrho_{\mathbb{H}^{n}}(x, z)\right) d \mu^{\prime}(z)=\cosh \left(\varrho_{\mathbb{H}^{n}}(b, x)\right) \cdot \int_{\mathbb{H}^{n}} \cosh \left(\varrho_{\mathbb{H}^{n}}(b, z)\right) d \mu^{\prime}(z) .
$$

Remark 2.2. The equality (2.12) provides an estimate for $\varrho_{\mathbb{H}^{n}}(b, x)$ in terms of the Leibniz hyperbolic function, more precisely, an immediate consequence of (2.12) is the equality:

$$
\begin{gathered}
2 \sinh \left(\frac{\varrho_{\mathbb{H}^{n}}(b, x)}{2}\right)^{2} \int_{\mathbb{H}^{n}} \cosh \left(\varrho_{\mathbb{H}^{n}}(b, z)\right) d \mu^{\prime}(z)= \\
=\int_{\mathbb{H}^{n}} \cosh \left(\varrho_{\mathbb{H}^{n}}(x, z)\right) d \mu^{\prime}(z)-\int_{\mathbb{H}^{n}} \cosh \left(\varrho_{\mathbb{H}^{n}}(b, z)\right) d \mu^{\prime}(z)
\end{gathered}
$$

Proof: The integrability condition satisfied by the measure $\mu^{\prime}$ allows to integrate both sides of the equality (2.11): by integration, the right-hand side of this equality goes to zero with $\eta$, because

$$
\eta \mapsto \int_{\mathbb{H}^{n}}\left[\cosh \left(\varrho_{\mathbb{H}^{n}}(\bar{c}(\eta), z)\right)-\cosh \left(\varrho_{\mathbb{H}^{n}}(\bar{c}(0), z)\right)\right] d \mu^{\prime}(z),
$$

defined on $]-\varepsilon, \varepsilon[$ attains its minimum when $\eta=0$, because $\bar{c}(0)=b$, thus

$$
\frac{1}{\eta} \int_{\mathbb{H}^{n}}\left[\cosh \left(\varrho_{\mathbb{H}^{n}}(\bar{c}(\eta), z)\right)-\cosh \left(\varrho_{\mathbb{H}^{n}}(\bar{c}(0), z)\right)\right] d \mu^{\prime}(z)
$$

goes to zero when $\eta \rightarrow 0$; then, integrating and making $\eta$ going to zero, we get:

$$
\int_{\mathbb{H}^{n}}\left(\cosh \left(\varrho_{\mathbb{H}^{n}}(x, z)\right)-\cosh \left(\varrho_{\mathbb{H}^{n}}(b, z) \cosh a\right)\right) d \mu^{\prime}(z)=0
$$

where $a=\varrho_{\mathbb{H}^{n}}(b, z)$, because $\frac{\cosh \eta-1}{\sinh \eta} \rightarrow 0$ when $\eta \rightarrow 0$. This ends the proof of the equality (2.12). Replacing $\cosh a$ by $\left(2 \sinh \left(\frac{a}{2}\right)^{2}+1\right)$, we immediately 
deduce the formula of the Remark which follows the Lemma 2.8.

It is now possible to show that the barycentre map $\bar{H}$ is an almostisometry: we shall prove that the map $H$ is close to the $\varepsilon$-Hausdorff approximation $h$, i.e. that the images of the same point $\bar{y}$ through $\bar{H}$ and $\tilde{h}$ are $A(n) \sqrt{\varepsilon}-$ close, where $A(n)$ a constant depending on the dimension $n$ only. This property is synthesized in the following

Lemma 2.9. Let $\varepsilon_{0}=\operatorname{inj}(X, g)$, then, for any $\varepsilon<\min \left(\frac{1}{64 n^{2}} \varepsilon_{0}, \frac{1}{72 c}, \frac{c-n}{64 c} \varepsilon_{0}\right)$ and for any $r$-approximation $\varrho$ of $\varrho_{\bar{M}}$ such that $r<\frac{\varepsilon}{2}$, one has:

$$
\sinh \left(\varrho_{\mathbb{H}^{n}}(\tilde{h}(\bar{y}), \bar{H}(\bar{y}))\right) \leq 4 n \sqrt{\frac{\varepsilon}{\varepsilon_{0}}}+6 \sqrt{\frac{n}{c-n}} \sqrt{\frac{\varepsilon}{\varepsilon_{0}}}+5 \sqrt{c \varepsilon}
$$

and thus $H$ is a $\varepsilon^{\prime}$-approximation, where $\varepsilon^{\prime}=4 n \sqrt{\frac{\varepsilon}{\varepsilon_{0}}}+6 \sqrt{\frac{n}{c-n}} \sqrt{\frac{\varepsilon}{\varepsilon_{0}}}+5 \sqrt{c \varepsilon}$

Remark 2.3. An immediate corollary of this Lemma is that, if $\varepsilon<\min \left(\frac{1}{64 n^{2}} \varepsilon_{0}, \frac{1}{144 n}\right)$ there is a choice of $c$ such that

$$
\varrho_{\mathbb{H}^{n}}(\tilde{h}(\bar{y}), \bar{H}(\bar{y}))<\left(\frac{4 n+6}{\sqrt{\varepsilon_{0}}}+5 \sqrt{2 n}\right) \sqrt{\varepsilon} .
$$

To prove this, it is sufficient to chose $c=2 n$, then $\varepsilon<\min \left(\frac{1}{64 n^{2}} \varepsilon_{0}, \frac{1}{144 n}\right)$ implies that $\varepsilon<\min \left(\frac{1}{64 n^{2}} \varepsilon_{0}, \frac{1}{72 c}, \frac{c-n}{64 c} \varepsilon_{0}\right)$ and thus

$$
\varrho_{\mathbb{H}^{n}}(\tilde{h}(\bar{y}), \bar{H}(\bar{y})) \leq 4 n \sqrt{\frac{\varepsilon}{\varepsilon_{0}}}+6 \sqrt{\frac{\varepsilon}{\varepsilon_{0}}}+5 \sqrt{n \varepsilon} .
$$

Proof. We use here the following notations:

- $d \nu_{\bar{y}}(\bullet)=e^{-c\left(1-\frac{3 \varepsilon}{2 \alpha}\right)^{-1} \varrho_{\mathbb{H}^{n}}(\tilde{h}(\bar{y}), \bullet)} d v_{\tilde{g}_{0}}(\bullet)$ and the corresponding Leibniz function

- $\widetilde{\mathcal{B}}_{\bar{y}}(\tilde{x})=\int_{\mathbb{H}^{n}} \cosh \varrho_{\mathbb{H}^{n}}(\tilde{x}, \tilde{z}) d \nu_{\bar{y}}(\tilde{z})$;

- $d \mu_{\bar{y}}=e^{-c \varrho(\bar{y}, \tilde{f}(\bullet))} d v_{\tilde{g}_{0}}(\bullet)$ and the corresponding Leibniz function

- $\mathcal{B}_{\bar{y}}(\tilde{x})=\int_{\mathbb{H}^{n}} \cosh \varrho_{\mathbb{H}^{n}}(\tilde{x}, \tilde{z}) d \mu_{\bar{y}}(\tilde{z})$.

By the sublemma 2.6 (resp. by definition), when $c>\frac{n}{1-\frac{3 \varepsilon}{\varepsilon_{0}}}, \tilde{h}(\bar{y})(\operatorname{resp} . \bar{H}(\bar{y}))$ is the barycentre of the measure $\nu_{\bar{y}}$ (resp. $\mu_{\bar{y}}$ ), the Lemma 2.8 then implies: 
Vol. LVI (2018) Volumes Comparison in the presence of a Gromov-Hausdorff ...113

$$
\left\{\begin{array}{l}
\mathcal{B}_{\bar{y}}(\tilde{h}(\bar{y}))=\mathcal{B}_{\bar{y}}(\bar{H}(\bar{y})) \cosh \left(\varrho_{\mathbb{H}^{n}}(\tilde{h}(\bar{y}), \bar{H}(\bar{y}))\right) \\
\widetilde{\mathcal{B}}_{\bar{y}}(\bar{H}(\bar{y}))=\widetilde{\mathcal{B}}_{\bar{y}}(\bar{h}(\bar{y})) \cosh \left(\varrho_{\mathbb{H}^{n}}(\tilde{h}(\bar{y}), \tilde{h}(\bar{y}))\right) .
\end{array}\right.
$$

By the inequality (2.2) of the Lemma 2.1 and by the condition 1 of the definition of $\varrho$, we have

$$
\begin{gathered}
e^{-c r} e^{-2 c \varepsilon} \widetilde{\mathcal{B}}_{\bar{y}}(\bar{H}(\bar{y}))-\mathcal{B}_{\bar{y}}(\bar{H}(\bar{y}))= \\
=\int_{\mathbb{H}^{n}} \cosh \varrho_{\mathbb{H}^{n}}(\bar{H}(\bar{y}), \tilde{z})\left[e^{-c\left[\left(1-\frac{3 \varepsilon}{2 \alpha}\right)^{-1} \varrho_{\mathbb{H}^{n}}(\tilde{h}(\bar{y}), \tilde{z})+2 \varepsilon+r\right]}-e^{-c \varrho(\bar{y}, \tilde{f}(\tilde{z}))}\right] d v_{\tilde{g}_{0}}(\tilde{z}) \leq 0 .
\end{gathered}
$$

Plugging this inequality in (2.13) we get:

$$
\begin{aligned}
& \mathcal{B}_{\bar{y}}(\tilde{h}(\bar{y}))=\mathcal{B}_{\bar{y}}(\bar{H}(\bar{y})) \cosh \left(\varrho_{\mathbb{H}^{n}}(\tilde{h}(\bar{y}), \bar{H}(\bar{y}))\right) \geq \\
& \geq e^{-c r} e^{-2 c \varepsilon} \widetilde{\mathcal{B}}_{\bar{y}}(\bar{H}(\bar{y})) \cosh \left(\varrho_{\mathbb{H}^{n}}(\tilde{h}(\bar{y}), \bar{H}(\bar{y}))\right) \geq \\
& \geq e^{-c(r+2 \varepsilon)} \widetilde{\mathcal{B}}_{\bar{y}}(\tilde{h}(\bar{y})) \cosh ^{2}\left(\varrho_{\mathbb{H}^{n}}(\tilde{h}(\bar{y}), \bar{H}(\bar{y}))\right) .
\end{aligned}
$$

Using the definition of $\mathcal{B}_{\bar{y}}$ and of $\widetilde{\mathcal{B}}_{\bar{y}}$, the inequality (2.1) of the Lemma 2.1 and by the condition 1 of the definition of $\varrho$, we deduce from the last inequality:

$$
\begin{gathered}
\int_{\mathbb{H}^{n}} \cosh \left(\varrho_{\mathbb{H}^{n}}(\tilde{h}(\bar{y}), \tilde{z})\right) e^{-c\left[\left(1-\frac{3 \varepsilon}{\varepsilon_{0}}\right) \varrho_{\mathbb{H}^{n}}(\tilde{h}(\bar{y}), \tilde{z})-2 \varepsilon-r\right]} d v_{\tilde{g}_{0}}(\tilde{z}) \geq \\
\geq e^{-c(r+2 \varepsilon)}\left[\int_{\mathbb{H}^{n}} \cosh \left(\varrho_{\mathbb{H}^{n}}(\tilde{h}(\bar{y}), \tilde{z})\right) e^{-c\left(1-\frac{3 \varepsilon}{2 \alpha}\right)^{-1} \varrho_{\mathbb{H}^{n}}(\tilde{h}(\bar{y}), \tilde{z})} d v_{\tilde{g}_{0}}(\tilde{z})\right] . \\
\cdot\left(1+\sinh ^{2}\left(\varrho_{\mathbb{H}^{n}}(\tilde{h}(\bar{y}), \bar{H}(\bar{y}))\right)\right)
\end{gathered}
$$

Let $\omega_{n}=\operatorname{Vol}\left(\mathbb{S}^{n}, c a n\right)$. Integrating with respect to the polar (exponential) coordinates of $\mathbb{H}^{n}$ centered at $\tilde{h}(\bar{y}$ ) (for a definition and properties, see the proof of the Lemma 2.3) the last inequality writes:

$$
\begin{gathered}
\omega_{n} \int_{0}^{+\infty} \cosh t(\sinh t)^{n-1} e^{-c\left(1-\frac{3 \varepsilon}{\varepsilon_{0}}\right) t} d t \geq \\
\geq e^{-2 c(2 \varepsilon+r)} \omega_{n}\left(\int_{0}^{+\infty} \cosh t(\sinh t)^{n-1} e^{-c\left(1-\frac{3 \varepsilon}{2 \alpha}\right)^{-1} t} d t\right) . \\
\cdot\left(1+\sinh ^{2}\left(\varrho_{\mathbb{H}^{n}}(\tilde{h}(\bar{y}), \bar{H}(\bar{y}))\right)\right)
\end{gathered}
$$


or, equivalently, when integrating by parts:

$1+\sinh ^{2}\left(\varrho_{\mathbb{H} n}(\tilde{h}(\bar{y}), \bar{H}(\bar{y}))\right) \leq e^{2 c(2 \varepsilon+r)}\left(1-\frac{3 \varepsilon}{\varepsilon_{0}}\right)\left(1-\frac{3 \varepsilon}{2 \alpha}\right) \cdot \frac{J_{n}\left(c\left(1-\frac{3 \varepsilon}{\varepsilon_{0}}\right)\right)}{J_{n}\left(c\left(1-\frac{3 \varepsilon}{2 \alpha}\right)^{-1}\right)}$

where $J_{n}(\beta)=\int_{0}^{+\infty}(\sinh t)^{n} e^{-\beta t} d t$.

We now use the

Remark 2.4. For every $\beta>n$ one has

$$
J_{n}(\beta)=\frac{n !}{\prod_{i=0}^{n}(\beta+n-2 i)}
$$

Proof of this Remark: Integrating by parts, we get, for $n \geq 1$,

$$
\int_{0}^{+\infty}(\sinh t)^{n} e^{-\beta t} d t=\frac{n}{\beta} \int_{0}^{+\infty} \cosh t(\sinh t)^{n-1} e^{-\beta t} d t
$$

and, integrating one more time by parts, we have, for $n \geq 2$ :

$$
\int_{0}^{+\infty}(\sinh t)^{n} e^{-\beta t} d t=\frac{n}{\beta^{2}} \int_{0}^{+\infty}\left[(\sinh t)^{n}+(n-1) \cosh ^{2} t(\sinh t)^{n-2}\right] e^{-\beta t} d t
$$

from which we deduce:

$$
J_{n}(\beta)=\frac{n(n-1)}{(\beta+n)(\beta-n)} J_{n-2}(\beta) .
$$

Noticing that $J_{0}(\beta)=\frac{1}{\beta}$ and $J_{1}(\beta)=\frac{1}{(\beta-1)(\beta+1)}$, the last inequality provides, by iteration, the formula (2.15).

As $c$ may be any real number strictly greater than $\frac{n}{1-\frac{3 \varepsilon}{\varepsilon_{0}}}$, defining $\varepsilon^{\prime}$ by $\varepsilon^{\prime}=\frac{c}{n}\left(1-\frac{3 \varepsilon}{\varepsilon_{0}}\right)-1, \varepsilon^{\prime}$ may be any strictly positive number, and have $c=\frac{n\left(1+\varepsilon^{\prime}\right)}{1-\frac{3 \varepsilon}{\varepsilon_{0}}}$.

Plugging this and the equality (2.15) in the formula (2.14), we get:

$$
\begin{gathered}
1+\sinh ^{2}\left(\varrho_{\mathbb{H}^{n}}(\tilde{h}(\bar{y}), \bar{H}(\bar{y}))\right) \leq \\
\leq e^{2 c(2 \varepsilon+r)} \cdot \frac{\left(1-\frac{3 \varepsilon}{\varepsilon_{0}}\right)\left(1-\frac{3 \varepsilon}{2 \alpha}\right) \prod_{i=0}^{n}\left(\frac{n\left(1+\varepsilon^{\prime}\right)}{\left(1-\frac{3 \varepsilon}{\varepsilon_{0}}\right)\left(1-\frac{3 \varepsilon}{2 \alpha}\right)}+n-2 i\right)}{\prod_{i=0}^{n}\left(n+n \varepsilon^{\prime}+n-2 i\right)}
\end{gathered}
$$


Vol. LVI (2018) Volumes Comparison in the presence of a Gromov-Hausdorff ...115

$$
\begin{gathered}
\leq \frac{e^{2 c(2 \varepsilon+r)}}{\left(1-\frac{3 \varepsilon}{\varepsilon_{0}}\right)^{n}\left(1-\frac{3 \varepsilon}{2 \alpha}\right)^{n}} \cdot\left(\frac{n\left(1+\epsilon^{\prime}\right)-n\left(1-\frac{3 \varepsilon}{\varepsilon_{0}}\right)\left(1-\frac{3 \varepsilon}{2 \alpha}\right)}{n \varepsilon^{\prime}}\right) . \\
\quad \prod_{i=0}^{n}\left(\frac{n\left(1+\varepsilon^{\prime}\right)+(n-2 i)\left(-3 \varepsilon\left(\frac{1}{\varepsilon_{0}}+\frac{1}{2 \alpha}-\frac{3 \varepsilon}{2 \alpha \varepsilon_{0}}\right)\right)}{2 n+n \varepsilon^{\prime}-2 i}\right) \\
\quad \leq \frac{e^{2 c(2 \varepsilon+r)}}{\left(1-\frac{3 \varepsilon}{\varepsilon_{0}}\right)^{n}\left(1-\frac{3 \varepsilon}{2 \alpha}\right)^{n}} \cdot\left(1+3 \frac{\varepsilon}{\varepsilon^{\prime}}\left(\frac{1}{\varepsilon_{0}}+\frac{1}{2 \alpha}\right)\right) . \\
\cdot \prod_{i=1}^{n}\left(1+\frac{2 i-n}{n\left(1+\varepsilon^{\prime}\right)-(2 i-n)}\left[3 \varepsilon\left(\frac{1}{\varepsilon_{0}}+\frac{1}{2 \alpha}-\frac{3 \varepsilon}{2 \alpha \varepsilon_{0}}\right)\right]\right) .
\end{gathered}
$$

Recalling that $\alpha$ is chosen to be $\alpha=\frac{\varepsilon_{0}}{3}$, we get:

$$
\begin{gathered}
1+\sinh ^{2}\left(\varrho_{\mathbb{H}^{n}}(\tilde{h}(\bar{y}), \bar{H}(\bar{y}))\right) \leq \\
\leq \frac{e^{2 c(2 \varepsilon+r)}}{\left(1-\frac{3 \varepsilon}{\varepsilon_{0}}\right)^{n}\left(1-\frac{3 \varepsilon}{2 \alpha}\right)^{n}}\left(1+8 \frac{\varepsilon}{\varepsilon^{\prime} \varepsilon_{0}}\right)\left(1+4(n-2) \frac{\varepsilon}{\varepsilon_{0}}\right)^{n} .
\end{gathered}
$$

In order to explicit this upper bound, we recall the following inequalities, that we already settled in the [7] (2013):

(a) $e^{x} \leq 1+x e^{x}$ for every $x \in \mathbb{R}^{+}$;

(b) $(1+x)^{n} \leq \frac{1}{(1-x)^{n}} \leq \frac{1}{1-n x} \leq 1+2 n x$ for every $x \in\left[0, \frac{1}{2 n}[\right.$

(c) $(1+x)(1+y)(1+z)(1+u)(1+v) \leq\left(1+\frac{x+y+z+u+v}{5}\right)^{5} \leq 1+2(x+y+z+$ $u+v)$ for every $(x, y, z, u, v) \in\left(\mathbb{R}^{+}\right)^{5}$ such that $x+y+z+u+v<\frac{1}{2}$,

where (c) is deduced from (b) and from the fact that, if the $\lambda_{i}$ 's are positive, then $\left(\frac{1}{n} \sum_{i=1}^{n} \lambda_{i}\right)^{n} \geq \prod_{i=1}^{n} \lambda_{i}$.

Applying (a), and recalling that $\varepsilon<\frac{1}{72 c}$, we get

- $e^{2 c(2 \varepsilon+r)}<e^{5 c \varepsilon}<1+6 c \varepsilon$

Applying (b) and recalling that, by the assumptions of the Lemma $2.9, \frac{\varepsilon}{\varepsilon_{0}}<$ $\frac{1}{64 n^{2}}$, we get

- $\frac{1}{\left(1-\frac{3 \varepsilon}{\varepsilon_{0}}\right)^{n}} \leq 1+6 n \frac{\varepsilon}{\varepsilon_{0}}$

- $\frac{1}{\left(1-\frac{9 \varepsilon}{2 \varepsilon_{0}}\right)^{n}} \leq 1+9 n \frac{\varepsilon}{\varepsilon_{0}}$ 
- $\left(1+4(n-2) \frac{3 \varepsilon}{\varepsilon_{0}}\right)^{n} \leq 1+8 n(n-2) \frac{\varepsilon}{\varepsilon_{0}}$.

Now, as $c=\frac{n\left(1+\varepsilon^{\prime}\right)}{1-\frac{3 \varepsilon}{\varepsilon_{0}}}$, a direct computation gives:

$$
\varepsilon^{\prime}=\frac{c-n}{n}\left(1-3 \frac{c}{c-n} \frac{\varepsilon}{\varepsilon_{0}}\right)
$$

and thus

$$
\left(1+8 \frac{\varepsilon}{\varepsilon^{\prime} \varepsilon_{0}}\right)=\left(1+8 \frac{\frac{n}{c-n} \frac{\varepsilon}{\varepsilon_{0}}}{1-3 \frac{c}{c-n} \frac{\varepsilon}{\varepsilon_{0}}}\right) \leq 1+9 \frac{n}{c-n} \frac{\varepsilon}{\varepsilon_{0}} .
$$

Plugging all these estimates in the formula (2.16) we get:

$$
\begin{gathered}
1+\sinh ^{2}\left(\varrho_{\mathbb{H}^{n}}(\tilde{h}(\bar{y}), \bar{H}(\bar{y}))\right) \leq \\
\leq(1+6 c \varepsilon)\left(1+6 n \frac{\varepsilon}{\varepsilon_{0}}\right)\left(1+9 n \frac{\varepsilon}{\varepsilon_{0}}\right)\left(1+8 n(n+2) \frac{\varepsilon}{\varepsilon_{0}}\right)\left(1+\frac{n}{c-n} \frac{\varepsilon}{\varepsilon_{0}}\right) .
\end{gathered}
$$

Noticing that

$$
6 c \varepsilon+6 n \frac{\varepsilon}{\varepsilon_{0}}+9 n \frac{\varepsilon}{\varepsilon_{0}}+8 n(n+2) \frac{\varepsilon}{\varepsilon_{0}}+9 \frac{n}{c-n} \frac{\varepsilon}{\varepsilon_{0}} \leq 1
$$

(by the assumptions of the Lemma 2.9 and the fact $c>n$ ), we may apply the formula (c), which gives:

$$
\begin{aligned}
\sinh ^{2}\left(\varrho_{\mathbb{H}^{n}}(\tilde{h}(\bar{y}), \bar{H}(\bar{y}))\right) & \leq 2\left(6 c \varepsilon+6 n \frac{\varepsilon}{\varepsilon_{0}}+9 n \frac{\varepsilon}{\varepsilon_{0}}+8 n(n+2) \frac{\varepsilon}{\varepsilon_{0}}+9 \frac{n}{c-n} \frac{\varepsilon}{\varepsilon_{0}}\right) \\
\leq & 16 n^{2} \frac{\varepsilon}{\varepsilon_{0}}+18 \frac{n}{c-n} \frac{\varepsilon}{\varepsilon_{0}}+12 c \varepsilon
\end{aligned}
$$

we conclude by taking the square root of this inequality and noticing that $\varrho \leq \sinh \varrho$.

\section{The regularization and the jacobian estimation of the (regularized) barycentre map}

Using the regularization of the distance function, it is possible to prove the Theorem 1.3 of this paper, defining the regularized barycentre as the point $\tilde{x}_{r}$ where the function $\mathcal{B}_{\bar{y}}^{r}:\left(\mathbb{H}^{n}, \tilde{g}_{0}\right) \rightarrow \mathbb{R}^{+}$:

$$
\mathcal{B}_{\bar{y}}^{r}(\tilde{x})=\int_{\mathbb{H}^{n}} \cosh \varrho_{\mathbb{H}^{n}}(\tilde{x}, \tilde{z}) e^{-c \varrho_{r}(\bar{y}, \tilde{f}(\tilde{z}))} d v_{\tilde{g}_{0}}(\tilde{z})
$$


Vol. LVI (2018) Volumes Comparison in the presence of a Gromov-Hausdorff ...117

attains its minimum, where $\varrho_{r}$ is the regularized approximate distance, (see [4] (1977)) defined as follow.

Let $\phi:\left[0,+\infty\left[\right.\right.$ be a $C^{\infty}$ function (mollifier) such that

1. $\phi=1$ in a neighbourhood of 0 ;

2. $\phi(t)=0 \quad \forall t \geq 1$;

3. $\phi^{\prime}(t) \leq 0 \quad \forall t \in[0,+\infty[$.

Now, let $i(\bar{M}, \bar{g})$ be the injectivity radius of $(\bar{M}, \bar{g})$, for $r<i(\bar{M}, \bar{g})$ we define the function $\phi_{r}$ by:

$$
\phi_{r}(t)=\frac{\phi\left(\frac{t}{r}\right)}{\operatorname{Vol}\left(\mathbb{S}^{n-1}, \operatorname{can}\right) \int_{0}^{r} \phi\left(\frac{s}{r}\right) s^{n-1} d s}=\frac{\frac{1}{r^{n}} \phi\left(\frac{t}{r}\right)}{\operatorname{Vol}\left(\mathbb{S}^{n-1}, \text { can }\right) \int_{0}^{1} \phi(t) t^{n-1} d t}
$$

The following properties have been established when $\varrho$ is any approximation of $\varrho_{\bar{M}}$ which satisfies the above properties 1., 2. and 3. . As $\varrho_{r}$ is such an approximation it immediately comes that $\mathcal{B}_{\bar{y}}^{r}$ is well defined for every $c>\frac{n}{1-\frac{3 \varepsilon}{\varepsilon_{0}}}$, is continuous, strictly convex and goes to infinity when the geodesic distance from a selected point goes to infinity (see Lemma 2.2). Thus $\bar{H}_{r}(\bar{y})$ is well defined as the point where $\mathcal{B}_{\bar{y}}^{r}$ attains its minimum. Thus $\bar{H}_{r}$ is the barycentre of the measure $\mu_{\bar{y}}^{r}=e^{-c \varrho_{r}(\bar{y}, \tilde{f}(\bullet))} d v_{\tilde{g}_{0}}$, where this notion of barycentre has been defined in the Lemma 2.2. We may thus substitute $\varrho_{r}$ and $\varrho$ in the Lemmas 2.4 and 2.8. Applying these Lemmas in the case $\varrho=\varrho_{r}$ we get the following properties for $H_{r}$ :

Lemma 3.1. For any $c>\frac{n}{1-\frac{3 \varepsilon}{\varepsilon_{0}}}$, the regularized barycentre map $\bar{H}_{r}$ is well defined, moreover

1. it is an equivariant map, i.e. for every $\gamma \in \operatorname{Isom}\left(\bar{M}, g_{\bar{M}}\right)$ one has

$$
\bar{H}_{r} \circ \gamma=\bar{r}(\gamma)^{-1} \circ \bar{H}_{r} .
$$

2. Let $\varepsilon_{0}$ the injectivity radius of $\left(\mathbb{H}^{n} / \Lambda, g_{0}\right)$, then, for every $\bar{y} \in \bar{M}$, for any $\varepsilon<\min \left(\frac{1}{64 n^{2}}, \frac{c-n}{64 c}, \frac{1}{72 c \varepsilon_{0}}\right) \cdot \varepsilon_{0}$ and for any $r<\min (\varepsilon, \operatorname{inj}(\bar{M}, \bar{g}))$,

$$
\varrho_{\mathbb{H}^{n}}\left(\bar{H}_{r}(\bar{y}), \tilde{h}(\bar{y})\right) \leq\left(4 n+5 \sqrt{\frac{n}{c-n}}\right) \sqrt{\frac{\varepsilon}{\varepsilon_{0}}}+4 \sqrt{c \varepsilon},
$$

and thus $\bar{H}_{r}$ is also a $\left[\left(4 n+5 \sqrt{\frac{n}{c-n}}\right) \sqrt{\frac{\varepsilon}{\varepsilon_{0}}}+4 \sqrt{c \varepsilon}\right]$-Hausdorff-approximation which is an almost inverse of $\tilde{f}$. 
Moreover the regularity of $\varrho_{r}$ implies the

Lemma 3.2. For every $c>\frac{n}{1-\frac{3 \varepsilon}{\varepsilon_{0}}}$, the regularized barycentre map $\bar{H}_{r}$ is $C^{1}\left(\bar{M}, \mathbb{R}^{n}\right)$ (and, in fact $\left.C^{\infty}\right)$.

Proof. As $\mathbb{H}^{n}$ is diffeomorphic to $\mathbb{R}^{n}$, we fix a global system of coordinates $\tilde{x} \mapsto\left(\tilde{x}_{1}, \tilde{x}_{2} \ldots, \tilde{x}_{n}\right)$ which is a diffeomorphism $\mathbb{H}^{n} \rightarrow \mathbb{R}^{n}$; the map $\Phi: \mathbb{H}^{n} \times$ $\bar{M} \rightarrow \mathbb{R}^{n}$, whose components are defined by:

$$
\begin{aligned}
& \Phi_{i}(\tilde{x}, \bar{y})=\left.\int_{\mathbb{H}^{n}} d\left(\cosh \varrho_{\mathbb{H}^{n}}\right)\right|_{(\tilde{x}, \tilde{z})}\left(\frac{\partial}{\partial \tilde{x}_{i}}\right) e^{-c \varrho_{r}(\bar{y}, \tilde{f}(\tilde{z}))} d v_{\tilde{g}_{0}}(\tilde{z})= \\
= & \int_{\mathbb{H}^{n}} \sinh \varrho_{\mathbb{H}^{n}}(\tilde{x}, \tilde{z})\left\langle\nabla \varrho_{\mathbb{H}^{n}}(\tilde{x}, \tilde{z}), \frac{\partial}{\partial \tilde{x}^{i}}\right\rangle e^{-c \varrho_{r}(\bar{y}, \tilde{f}(\tilde{z}))} d v_{\tilde{g}_{0}}(\tilde{z}),
\end{aligned}
$$

is such that $\Phi(\tilde{x}, \bar{y})=\mathbf{0}_{\mathbb{R}^{n}}$ if and only if $\tilde{x}=\bar{H}_{r}(\bar{y})$ by the property of the barycentre (Lemma 2.4). We prove that $\Phi$ verifies the assumptions of the Implicit Function Theorem of $U$. Dini in an open neighbourhood $U \times$ $V \subset \mathbb{H}^{n} \times \bar{M}$ of any point $\left(\tilde{x}_{0}, \bar{y}_{0}\right) \in \mathbb{H}^{n} \times \bar{M}$. Then there shall exist a neighbourhood $V^{\prime} \subset V$ of $\bar{y}_{0}$ in $\bar{M}$, a $C^{1} \operatorname{map} h_{r}: V^{\prime} \rightarrow \mathbb{H}^{n}$ and a neighbourhood $\Omega$ of $\left(\tilde{x}_{0}, \bar{y}_{0}\right)$ in $\mathbb{H}^{n} \times \bar{M}$ such that

$$
\left.\begin{array}{c}
(\tilde{x}, \bar{y}) \in \Omega \\
\text { and } \\
\Phi(\tilde{x}, \bar{y})=0
\end{array}\right\} \Longleftrightarrow\left\{\begin{array}{c}
\bar{y} \in V^{\prime} \\
\text { and } \\
\tilde{x}=h_{r}(\bar{y}) .
\end{array}\right.
$$

As a consequence, the uniqueness of the barycentre (i.e. of the solution of the equation $\Phi(\tilde{x}, \bar{y})=\mathbf{0}_{\mathbb{R}^{n}}$ for a fixed $\left.\bar{y} \in \bar{M}\right)$ implies that the locally defined map $h_{r}$ given by the implicit function theorem coincides with the restriction of the globally defined $\bar{H}_{r}$ to $V^{\prime}$. As $h_{r}$ is $C^{1}$ in the open neighbourhood $V^{\prime}$ of $\bar{y}_{0}, \bar{H}_{r}$ is also $C^{1}$ on $V^{\prime}$; as the point $\bar{y}_{0}$ can be chosen to be any point in $\bar{M}, \bar{H}_{r}$ is everywhere $C^{1}$ on $\bar{M}$.

Now, let us prove that we can apply the implicit function theorem to the $\operatorname{map} \Phi$.

Step 1. The map $\Phi$ is $C^{1}$ with respect to the couple of variables $(\tilde{x}, \bar{y})$. To show this, consider the $i$-th $\Phi_{i}$ component of $\Phi$, (see (3.1)): we shall prove that its derivative with respect to $\tilde{x}_{j}$ exists and writes

$$
\begin{gathered}
\left.\frac{\partial \Phi_{i}}{\partial \tilde{x}_{j}}\right|_{(\tilde{x}, \bar{y})}=\int_{\mathbb{H}^{n}} \frac{\partial}{\partial \tilde{x}_{j}}\left(\left.d\left(\cosh \varrho_{\mathbb{H}^{n}}\right)\right|_{(\tilde{x}, \tilde{z})}\left(\frac{\partial}{\partial \tilde{x}_{i}}\right)\right) e^{-c \varrho_{r}(\bar{y}, \tilde{f}(\tilde{z}))} d v_{\tilde{g}_{0}}(\tilde{z})= \\
=\left.\int_{\mathbb{H}^{n}} D d\left(\cosh \varrho_{\mathbb{H}^{n}}\right)\right|_{(\tilde{x}, \tilde{z})} e^{-c \varrho_{r}(\bar{y}, \tilde{f}(\tilde{z}))} d v_{\tilde{g}_{0}}(\tilde{z})
\end{gathered}
$$


Vol. LVI (2018) Volumes Comparison in the presence of a Gromov-Hausdorff ...119

$$
=\int_{\mathbb{H}^{n}} \cosh \varrho_{\mathbb{H}^{n}}(\tilde{x}, \tilde{z}) \tilde{g}_{0}\left(\frac{\partial}{\partial \tilde{x}^{i}}, \frac{\partial}{\partial \tilde{x}^{j}}\right) e^{-c \varrho_{r}(\bar{y}, \tilde{f}(\tilde{z}))} d v_{\tilde{g}_{0}}(\tilde{z}),
$$

where the second inequality comes from (3.1). Let $\tilde{x}_{0}$ (resp. $\left.\bar{y}_{0}\right)$ be any point of $\mathbb{H}^{n}$ (resp. of $\left.\bar{M}\right)$ and let $B_{\mathbb{H}^{n}}\left(\tilde{x}_{0}, 1\right)$ (resp. $B_{\bar{M}}\left(\bar{y}_{0}, 1\right)$ be the unit ball centered at $\tilde{x}_{0}$ (resp. at $\left.\bar{y}_{0}\right)$. The triangle-inequality, the Lemma 2.1 and the property $\left|\varrho_{r}-\varrho_{\bar{M}}\right| \leq r$ of the regularized distance (point 1. above) give

$$
\begin{aligned}
& \left.D d\left(\cosh \varrho_{\mathbb{H}^{n}}\right)\right|_{(\tilde{x}, \tilde{z})}\left(\frac{\partial}{\partial \tilde{x}_{j}}, \frac{\partial}{\partial \tilde{x}_{i}}\right) e^{-c \varrho_{r}(\bar{y}, \tilde{f}(\tilde{z}))} \leq \cosh \varrho_{\mathbb{H}^{n}}(\tilde{x}, \tilde{z}) e^{-c \varrho_{r}(\bar{y}, \tilde{f}(\tilde{z}))} \\
& \leq e^{c(1+r+3 \varepsilon)} \cosh \left[1+\varrho_{\mathbb{H}^{n}}\left(\tilde{x}_{0}, \tilde{z}\right)\right] e^{-c\left(1-\frac{3 \varepsilon}{\varepsilon_{0}}\right) \varrho_{\mathbb{H}^{n}}\left(\tilde{h}\left(\bar{y}_{0}\right), \tilde{z}\right)}
\end{aligned}
$$

The right-hand term of this inequality is a function independent on $\tilde{x}$ and on $\bar{y}$ which is $d v_{\tilde{g}_{0}}(\tilde{z})$-integrable when $c>\frac{n}{1-\frac{3 \varepsilon}{\varepsilon_{0}}}$. Thus the Lebesgue Dominate derivability and continuity theorems prove the equality (3.2) and the continuity of $\frac{\partial \Phi}{\partial \tilde{x}_{i}}$ with respect to the couple $(\tilde{x}, \bar{y})$ We consider, now, a local chart $\bar{y} \rightarrow\left(\bar{y}_{1}, \ldots, \bar{y}_{n}\right)$ in the manifold $\bar{M}$. In order to prove that the integral and the partial derivative commute, i.e. that the partial derivatives with respect to the $\bar{y}$-variables are:

$$
\begin{aligned}
\left.\frac{\partial \Phi_{i}}{\partial \bar{y}_{j}}\right|_{(\tilde{x}, \bar{y})} & =-c \int_{\mathbb{H}^{n}} \sinh \varrho_{\mathbb{H}^{n}}(\tilde{x}, \tilde{z})\left\langle\nabla \varrho_{\mathbb{H}^{n}}(\tilde{x}, \tilde{z}), \frac{\partial}{\partial \tilde{x}_{i}}\right\rangle \\
\cdot & \left\langle\nabla \varrho_{r}(\bar{y}, \tilde{f}(\tilde{z})), \frac{\partial}{\partial \bar{y}_{j}}\right\rangle e^{-c \varrho_{r}(\bar{y}, \tilde{f}(\tilde{z}))} d v_{\tilde{g}_{0}}(\tilde{z}),
\end{aligned}
$$

let us fix again $\bar{y}_{0}$ in $\bar{M}$ and let $\bar{y}$ run in the geodesic unit ball $B_{\bar{M}}\left(\bar{y}_{0}, 1\right)$. The triangle-inequality and the fact that the norm of the gradient of the regularized distance is upper bounded: $\left\|\nabla \varrho_{r}\right\| \leq 1+c_{1} k^{2} r^{2}$ (point 3. above) give

$$
\begin{aligned}
& \left|\sinh \varrho_{\mathbb{H}^{n}}(\tilde{x}, \tilde{z})\left\langle\nabla \varrho_{\mathbb{H}^{n}}(\tilde{x}, \tilde{z}) \frac{\partial}{\partial \tilde{x}_{i}}\right\rangle\left\langle\nabla \varrho_{r}(\bar{y}, \tilde{f}(\tilde{z})), \frac{\partial}{\partial \bar{y}_{j}}\right\rangle e^{-c \varrho_{r}(\bar{y}, \tilde{f}(\tilde{z}))}\right| \leq \\
& \leq\left(1+c_{1} k^{2} r^{2}\right) e^{c(1+r+2 \varepsilon)} \cosh \left[1+\varrho_{\mathbb{H}^{n}}\left(\tilde{x}_{0}, \tilde{z}\right)\right] e^{-c\left(1-\frac{3 \varepsilon}{\varepsilon_{0}}\right) \varrho_{\mathbb{H}^{n}}\left(\tilde{h}\left(\bar{y}_{0}\right), \tilde{z}\right)} .
\end{aligned}
$$

The right-hand term is a function independent on $\tilde{x}$ and $\bar{y}$, whose integral converges when $c>\frac{n}{1-\frac{3 \varepsilon}{\varepsilon_{0}}}$. Then the Lebesgue Dominate Derivation Theorem applies and justifies the equality (3.4); the continuity of $\frac{\partial \Phi}{\partial \bar{y}_{i}}(\tilde{x}, \bar{y})$ with respect to $(\tilde{x}, \bar{y})$ is again a consequence of the Lebesgue 
Dominate Continuity Theorem.

In conclusion, all the derivatives $\frac{\partial \Phi}{\partial \tilde{x}_{i}}$ and all the derivatives $\frac{\partial \Phi}{\partial \bar{y}_{j}}$ exist and are continuous with respect to $(\tilde{x}, \bar{y})$, thus the map $\Phi$ is $C^{1}$.

Step 2. Let now $\bar{y}_{0}$ be any point in $\bar{M}$. In order to apply the Implicit Function Theorem, it remains to prove that $\left(\frac{\partial \Phi_{i}}{\partial \tilde{x}_{j}}(\tilde{x}, \bar{y})\right)_{i, j}$ is a invertible matrix.

By the equality (3.2)

$$
\frac{\partial \Phi_{i}}{\partial \tilde{x}_{j}}(\tilde{x}, \bar{y})=\left.\lambda(\tilde{x}, \bar{y}) \tilde{g}_{0}\right|_{\tilde{x}}\left(\frac{\partial}{\partial \tilde{x}_{i}}, \frac{\partial}{\partial \tilde{x}_{j}}\right)
$$

where $\lambda(\tilde{x}, \bar{y})=\int_{\mathbb{H}^{n}} \cosh \varrho_{\mathbb{H}^{n}}(\tilde{x}, \tilde{z}) e^{-c \varrho_{r}(\bar{y}, \tilde{f}(\tilde{z}))} d v_{\tilde{g}_{0}}(\tilde{z})$ is strictly positive and $\left.\tilde{g}_{0}\right|_{\tilde{x}}\left(\frac{\partial}{\partial \tilde{x}_{i}}, \frac{\partial}{\partial \tilde{x}_{j}}\right)$ is positive definite. We immediately deduce that $\frac{\partial \Phi_{i}}{\partial \tilde{x}_{j}}(\tilde{x}, \bar{y})$ is invertible.

We have now all the necessary tools to prove the main Theorem 1.3 of this Section:

Lemma 3.3. and end of the proof of the main Theorem 1.3:

Fix $c+1$, if $\varepsilon \leq \frac{1}{64 n^{2}} \min \left(\varepsilon_{0}, 1\right)$, then

(i)

$$
\sum_{i=1}^{n}\left\|d_{\bar{y}} \bar{H}_{r}\left(\mathbf{e}_{i}^{\prime}\right)\right\|^{2} \leq n(1+160 n(n+1)) \sqrt{\frac{\varepsilon}{\min \left(\varepsilon_{0}, 1\right)}}
$$

for any orthonormal basis $\left\{\mathbf{e}_{i}^{\prime}\right\}_{1 \leq i \leq n}$ of $T_{\bar{y}} \bar{M}$,

(ii) the Jacobian determinant of $\bar{H}_{r}$ is everywhere bounded above by

$$
\left((1+160 n(n+1)) \sqrt{\frac{\varepsilon}{\min \left(\varepsilon_{0}, 1\right)}}\right)^{\frac{n}{2}}
$$

Proof. For the sake of simplicity, we write:

$$
\tilde{\varrho}=\varrho_{\mathbb{H}^{n}} \quad d \tilde{z}=d v_{\tilde{g}_{0}} \quad \text { and } \quad d \mu_{\bar{y}}^{r}(\tilde{z})=e^{-c \varrho_{r}(\bar{y}, \tilde{f}(\tilde{z}))} d v_{\tilde{g}_{0}}(\tilde{z}) .
$$

In the computations all the differentials will be taken with respect to the first component (i.e. $\varrho_{\mathbb{H}^{n}}(\tilde{x}, \tilde{z})$ will be differentiated with respect to $\tilde{x}$ and $\varrho_{r}(\bar{y}, \tilde{f}(\tilde{z}))$ will be differentiated with respect to $\left.\bar{y}\right)$. Let us recall the Lemma 2.4 (where $\varrho_{r}$ may be substituted to $\varrho$ because $\varrho_{r}$ is a "good" approximation 
Vol. LVI (2018) Volumes Comparison in the presence of a Gromov-Hausdorff ...121

in the above sense) that the regularized barycentre map $\bar{H}_{r}$ is characterized by the implicit equation:

$$
\int_{\mathbb{H}^{n}} d\left[\cosh \tilde{\varrho}\left(\bar{H}_{r}(\bar{y}), \tilde{z}\right)\right](\mathbf{v}) e^{-c \varrho_{r}(\bar{y}, \tilde{f}(\tilde{z}))} d \tilde{z}=0
$$

for every $\mathbf{v} \in T_{\bar{H}(\bar{y})} \mathbb{H}^{n}$.

Let $m \mapsto\left(\tilde{x}_{1}(m), \tilde{x}_{2}(m), \ldots, \tilde{x}_{n}(m)\right)$ be a coordinate system which is a diffeomorphism $\mathbb{H}^{n} \rightarrow \mathbb{R}^{n}$. Let, as in (3.1), $\Phi_{i}: \mathbb{H}^{n} \times \bar{M} \rightarrow \mathbb{R}$ be defined by:

$$
\Phi_{i}(\tilde{x}, \bar{y})=\int_{\mathbb{H}^{n}} d[\cosh \tilde{\varrho}(\tilde{x}, \tilde{z})]\left(\frac{\partial}{\partial \tilde{x}_{i}}\right) e^{-c \varrho_{r}(\bar{y}, \tilde{f}(\tilde{z}))} d \tilde{z}
$$

and $\Phi: \mathbb{H}^{n} \times \bar{M} \rightarrow \mathbb{R}^{n}$ by:

$$
\Phi(\tilde{x}, \bar{y})=\left(\phi_{1}(\tilde{x}, \bar{y}), \ldots, \phi_{2}(\tilde{x}, \bar{y})\right) .
$$

It has been proved in the Lemma 3.2 that $\Phi$ is $C^{1}$ and, from the computation of $\frac{\partial \phi_{i}}{\partial \tilde{x}_{j}}$ and $\frac{\partial \phi_{i}}{\partial \bar{y}_{j}}$ given by the formulas (3.2) and (3.4), we get, for every $\mathbf{w} \in$ $T_{\tilde{x}} \mathbb{H}^{n}$ and every $\mathbf{u} \in T_{\bar{y}} \bar{M}$ :

$$
\left\{\begin{array}{l}
d_{1} \phi_{i}(\mathbf{w})=\int_{\mathbb{H}^{n}} \cosh (\tilde{\varrho}(\tilde{x}, \tilde{z})) \tilde{g}_{0}\left(\frac{\partial}{\partial \tilde{x}_{i}}, \mathbf{w}\right) d \mu_{\bar{y}}^{r}(\tilde{z}) \\
d_{2} \phi_{i}(\mathbf{u})=-c \int_{\mathbb{H}^{n}} d[\cosh (\tilde{\varrho}(\tilde{x}, \tilde{z}))]\left(\frac{\partial}{\partial \tilde{x}_{i}}\right) \bar{g}\left(\nabla \varrho_{r}(\bar{y}, \tilde{f},(\tilde{z})), \mathbf{u}\right) d \mu_{\bar{y}}^{r}(\tilde{z})
\end{array}\right.
$$

where $d_{1}$ and $d_{2}$ denote the derivatives with respect to the parameters $\tilde{x}$ and $\bar{y}$ respectively. As $\bar{H}_{r}$ is regular (by Lemma 3.2 ), we may derive the equality $\Phi\left(\bar{H}_{r}(\bar{y}), \bar{y}\right)=0$ and get:

$$
\left.d_{1} \phi_{i}\right|_{\left(\bar{H}_{r}(\bar{y}), \bar{y}\right)}\left(d_{\bar{y}} \bar{H}_{r}(\mathbf{u})\right)+\left.d_{2} \phi_{i}\right|_{\left(\bar{H}_{r}(\bar{y}), \bar{y}\right)}(\mathbf{u})=0 .
$$

for every $i \in\{1, \ldots, n\}$. Using the equalities (3.7), we get:

$$
\begin{gathered}
\int_{\mathbb{H}^{n}} \cosh \left[\tilde{\varrho}\left(\bar{H}_{r}(\bar{y}), \tilde{z}\right)\right] \tilde{g}_{0}\left(\frac{\partial}{\partial \tilde{x}_{i}}, d_{\bar{y}} \bar{H}_{r}(\mathbf{u})\right) d \mu_{\bar{y}}^{r}(\tilde{z})- \\
-c \int_{\mathbb{H}^{n}} d\left[\cosh \tilde{\varrho}\left(\bar{H}_{r}(\bar{y}), \tilde{z}\right)\right]\left(\frac{\partial}{\partial \tilde{x}_{i}}\right) \bar{g}\left(\nabla \varrho_{r}(\bar{y}, \tilde{f}(\tilde{z})), \mathbf{u}\right) d \mu_{\bar{y}}^{r}(\tilde{z})=0 .
\end{gathered}
$$

Thus we get, for every $\mathbf{v} \in T_{\bar{H}_{r}(\bar{y})} \mathbb{H}^{n}$ :

$$
\tilde{g}_{0}\left(d_{\bar{y}} \bar{H}_{r}(\mathbf{u}), \mathbf{v}\right) \int_{\mathbb{H}^{n}} \cosh \tilde{\varrho}\left(\bar{H}_{r}(\bar{y}), \tilde{z}\right) d \mu_{\bar{y}}^{r}(\tilde{z})=
$$


$=c \int_{\mathbb{H}^{n}} \tilde{g}_{0}\left(\nabla \tilde{\varrho}_{r}\left(\bar{H}_{r}(\bar{y}), \tilde{z}\right), \mathbf{v}\right) \cdot \bar{g}\left(\nabla \varrho_{r}(\bar{y}, \tilde{f}(\tilde{z})), \mathbf{u}\right) \sinh \tilde{\varrho}\left(\bar{H}_{r}(\bar{y}), \tilde{z}\right) d \mu_{\bar{y}}^{r}(\tilde{z})$.

Let $\mathbf{v}$ be a unit-vector colinear to $d_{\bar{y}} \bar{H}_{r}(\mathbf{u})$, then the Cauchy-Schwarz inequality gives:

$$
\begin{gathered}
\left\|d_{\bar{y}} \bar{H}_{r}(\mathbf{u})\right\|^{2} \cdot\left(\int_{\mathbb{H}^{n}} \cosh \tilde{\varrho}\left(\bar{H}_{r}(\bar{y}), \tilde{z}\right) d \mu_{\bar{y}}^{r}(\tilde{z})\right)^{2} \leq \\
\leq c^{2}\left(\int_{\mathbb{H}^{n}} \tilde{g}_{0}\left(\nabla \tilde{\varrho}\left(\bar{H}_{r}(\bar{y}), \tilde{z}\right), \mathbf{v}\right)^{2} \sinh \tilde{\varrho}\left(\bar{H}_{r}(\bar{y}), \tilde{z}\right) d \mu_{\bar{y}}^{r}(\tilde{z})\right) . \\
\cdot\left(\int_{\mathbb{H}^{n}} \bar{g}\left(\nabla \varrho_{r}(\bar{y}, \tilde{f}(\tilde{z}), \mathbf{u})\right)^{2} \sinh \tilde{\varrho}\left(\bar{H}_{r}(\bar{y}), \tilde{z}\right) d \mu_{\bar{y}}^{r}(\tilde{z})\right) .
\end{gathered}
$$

From the inequalities (2.1) and (2.2) of the Lemma 2.1 and from the first property of the regularized distance function, it comes

$$
\left(1-\frac{3 \varepsilon}{\varepsilon_{0}}\right) \tilde{\varrho}(\tilde{z}, \tilde{h}(\bar{y}))-2 \varepsilon-r \leq \varrho_{r}(\bar{y}, \tilde{f}(\tilde{z})) \leq\left(1-\frac{3 \varepsilon}{2 \alpha}\right)^{-1} \tilde{\varrho}(\tilde{z}, \tilde{h}(\bar{y}))+2 \varepsilon+r .
$$

As $\tilde{\varrho}(\tilde{h}(\bar{h}), \bar{H}(\bar{y})) \leq \delta$, where $\delta=\left(4 n+5 \sqrt{\frac{n}{c-n}}\right) \sqrt{\frac{\varepsilon}{\varepsilon_{0}}}+4 \sqrt{c} \sqrt{\varepsilon}$ by the Lemma 2.8 , the triangle inequality and the fact that $r$ is chosen such that $r<\varepsilon$ both imply:

$$
\left(1-\frac{3 \varepsilon}{\varepsilon_{0}}\right) \tilde{\varrho}\left(\bar{H}_{r}(\bar{y}), \tilde{z}\right)-2 \varepsilon+\delta+r \leq \varrho_{r}(\bar{y}, \tilde{f}(\tilde{z}))
$$

and, as $\varepsilon<\frac{\alpha}{5}$, we have $\left(1-\frac{3 \epsilon}{2 \alpha}\right)^{-1}<\frac{3}{2}$, and thus

$$
\varrho_{r}(\bar{y}, \tilde{f}(\tilde{z})) \leq\left(1-\frac{3 \varepsilon}{2 \alpha}\right)^{-1} \tilde{\varrho}\left(\bar{H}_{r}(\bar{y}), \tilde{z}\right)+\frac{3}{2} \delta+2 \varepsilon+r
$$

in fact, if $\varepsilon$ is small enough, one may suppose that $\left(1-\frac{3 \varepsilon}{2 \alpha}\right)^{-1} \leq 2$. Plugging these estimates in (3.8), we deduce that for every $\mathbf{u} \in T_{\bar{y}} \bar{M}$ and any $\mathbf{v} \in$ $T_{\bar{H}_{r}(\bar{y})} \mathbb{H}^{n} \simeq \mathbb{R}^{n}$ :

$$
\begin{aligned}
& \left\|d_{\bar{y}} \bar{H}_{r}(\mathbf{u})\right\|^{2} e^{-c(3 \delta+4 \varepsilon+2 r)}\left(\int_{\mathbb{H}^{n}} \cosh \left(\tilde{\varrho}\left(\bar{H}_{r}(\bar{y}), \tilde{z}\right)\right) e^{-c\left(1-\frac{3 \varepsilon}{2 \alpha}\right)^{-1} \tilde{\varrho}\left(\bar{H}_{r}(\bar{y}), \tilde{z}\right)} d \tilde{z}\right)^{2} \leq \\
& c^{2}\left(e^{c(2 \varepsilon+\delta+r)} \int_{\mathbb{H}^{n}} \tilde{g}_{0}\left(\nabla \tilde{\varrho}\left(\bar{H}_{r}(\bar{y}) \tilde{z}\right), \mathbf{v}\right)^{2} \sinh \left(\tilde{\varrho}\left(\bar{H}_{r}(\bar{y}), \tilde{z}\right)\right) e^{-c\left(1-\frac{3 \varepsilon}{\varepsilon_{0}}\right) \tilde{\varrho}\left(\bar{H}_{r}(\bar{y}), \tilde{z}\right)} d \tilde{z}\right) \\
& \cdot\left(e^{c(2 \varepsilon+\delta+r)} \int_{\mathbb{H}^{n}} \bar{g}\left(\nabla \varrho_{r}(\bar{y}, \tilde{f}(\tilde{z})), \mathbf{u}\right)^{2} \sinh \left(\tilde{\varrho}\left(\bar{H}_{r}(\bar{y}), \tilde{z}\right)\right) e^{-c\left(1-\frac{3 \varepsilon}{\varepsilon_{0}}\right) \tilde{\varrho}\left(\bar{H}_{r}(\bar{y}), \tilde{z}\right)} d \tilde{z}\right) .
\end{aligned}
$$


Vol. LVI (2018) Volumes Comparison in the presence of a Gromov-Hausdorff ...123

identifying the unit sphere of $\left(T_{\bar{H}_{r}(\bar{y})} \mathbb{H}^{n},\left.\tilde{g}_{0}\right|_{\bar{H}_{\bar{y}}}\right)$ with $\mathbb{S}^{n-1}$, we may now consider the polar coordinates

$$
\varphi\left\{\begin{array}{cl}
\mathbf{R}^{+} \times \mathbb{S}^{n-1} & \rightarrow \mathbb{H}^{n} \\
(t, \mathbf{w}) & \mapsto \exp _{\bar{H}_{r}(\bar{y})}(t \cdot \mathbf{w})
\end{array}\right.
$$

one has:

- $\tilde{\varrho}\left(\bar{H}_{r}(\bar{y}), \varphi(t, \mathbf{w})\right)=t$,

- $d \tilde{z}=\sinh ^{n-1} t d t d \vartheta(\mathbf{w})$ where $d \vartheta$ is the canonical measure on $\mathbb{S}^{n-1}$.

Writing the last inequality with respect to the polar coordinates (i.e. making $\tilde{z}=\varphi(t, \mathbf{w}))$, we get:

$$
\begin{aligned}
& e^{-c(3 \delta+4 \varepsilon+2 r)}\left\|d_{\bar{y}} \bar{H}_{r}(\mathbf{u})\right\|^{2}\left(\omega_{n-1} \int_{0}^{\infty} e^{-c\left(1-\frac{3 \varepsilon}{2 \alpha}\right)^{-1} t} \cosh t \cdot(\sinh t)^{n-1} d t\right)^{2} \\
& \leq c^{2} e^{2 c(2 \varepsilon+\delta+r)}\left(\int_{0}^{\infty}\left(\int_{\mathbb{S}^{n-1}} \tilde{g}_{0}(\mathbf{v}, \mathbf{w})^{2} d \vartheta(\mathbf{w})\right)(\sinh t)^{n} e^{-c\left(1-\frac{3 \varepsilon}{\varepsilon_{0}}\right) t} d t\right) . \\
& \cdot\left(\int_{\mathbb{H}^{n}} \bar{g}\left(\nabla \varrho_{r}(\bar{y}, \tilde{f}(\tilde{z}), \mathbf{u})\right)^{2} \sinh \left[\tilde{\varrho}\left(\bar{H}_{r}(\bar{y}), \tilde{z}\right)\right] e^{-c\left(1-\frac{3 \varepsilon}{\varepsilon_{0}}\right) \tilde{\varrho}\left(\bar{H}_{r}(\bar{y}), \tilde{z}\right)} d \tilde{z}\right) \cdot
\end{aligned}
$$

For any symmetric bilinear form $\mathcal{Q}$ in any Euclidean space of dimension $n$, it is a classical result that the trace of $\mathcal{Q}$ with respect to the scalar product satisfies:

$$
\operatorname{Trace}_{g_{0}}(\mathcal{Q})=\sum_{i=1}^{n} \mathcal{Q}\left(\mathbf{e}_{i}, \mathbf{e}_{i}\right)=\frac{n}{\operatorname{Vol} \mathbb{S}^{n-1}} \int_{\mathbb{S}^{n-1}} \mathcal{Q}(\mathbf{w}, \mathbf{w}) d \vartheta(\mathbf{w})
$$

where $\left\{\mathbf{e}_{i}\right\}_{1 \leq i \leq n}$ is an orthonormal basis of the Euclidean space.

As the vector $\mathbf{v}$ is fixed (see above), let us consider the symmetric bilinear form

$$
\left(\mathbf{w}, \mathbf{w}^{\prime}\right) \mapsto g_{0}(\mathbf{v}, \mathbf{w}) \cdot g_{0}\left(\mathbf{v}, \mathbf{w}^{\prime}\right)
$$

we deduce that

$$
\int_{\mathbb{S}^{n-1}} g_{0}(\mathbf{v}, \mathbf{w})^{2} d \vartheta(\mathbf{w})=\frac{\omega_{n-1}}{n} \sum_{i=1}^{n} g_{0}\left(\mathbf{v}, \mathbf{e}_{i}\right)^{2}=\frac{\omega_{n-1}}{n}\|\mathbf{v}\|^{2}=\frac{\omega_{n-1}}{n} .
$$

On the other hand, if $\left\{\mathbf{e}_{i}^{\prime}\right\}_{1 \leq i \leq n}$ is an orthonormal basis of $\left(T_{\bar{y}} \bar{M}, \bar{g}\right)$ we have

$$
\sum_{i=1}^{n} \bar{g}\left(\nabla \varrho_{r}(\bar{y}, \tilde{f}(\tilde{z})), \mathbf{e}_{i}^{\prime}\right)^{2}=\left\|\nabla \varrho_{r}(\bar{y}, \tilde{f}(\tilde{z}))\right\|^{2} \leq\left(1+c_{1} k^{2} r^{2}\right)^{2} .
$$


Let $\left\{\mathbf{e}_{i}^{\prime}\right\}_{1 \leq i \leq n}$ be a $\bar{g}$-orthonormal basis of $T_{\bar{y}} \bar{M}$, plugging these estimates in the inequality (3.9) we get, by summation

$$
\begin{gathered}
\left(\sum_{i=1}^{n}\left\|d_{\bar{y}} \bar{H}_{r}\left(\mathbf{e}_{i}^{\prime}\right)\right\|^{2}\right)\left(\omega_{n-1} \int_{0}^{\infty} \cosh t \cdot(\sinh t)^{n-1} e^{-c\left(1-\frac{3 \varepsilon}{2 \alpha}\right)^{-1} t} d t\right)^{2} \leq \\
\leq c^{2} e^{c(5 \delta+8 \varepsilon+4 r)}\left(\frac{\omega_{n-1}}{n} \int_{0}^{\infty}(\sinh t)^{n} e^{-c\left(1-\frac{3 \varepsilon}{\varepsilon_{0}}\right) t} d t\right) . \\
\cdot\left(1+c_{1} k^{2} r^{2}\right)\left(\omega_{n-1} \int_{0}^{\infty}(\sinh t)^{n} e^{-c\left(1-\frac{3 \varepsilon}{\varepsilon_{0}}\right) t} d t\right)
\end{gathered}
$$

and thus

$$
\begin{gathered}
\left(\sum_{i=1}^{n}\left\|d_{\bar{y}} \bar{H}_{r}\left(\mathbf{e}_{i}^{\prime}\right)\right\|^{2}\right) \leq \frac{e^{c(5 \delta+8 \varepsilon+4 r)}}{n} \cdot\left(1+c_{1} k^{2} r^{2}\right)^{2} \\
\cdot \frac{\left(c \int_{0}^{\infty}(\sinh t)^{n} e^{-c\left(1-\frac{3 \varepsilon}{\varepsilon_{0}}\right) t} d t\right)^{2}}{\left(\int_{0}^{\infty} \cosh t \cdot(\sinh t)^{n-1} e^{-c\left(1-\frac{3 \varepsilon}{2 \alpha}\right)^{-1} t} d t\right)^{2}} \\
=e^{c(5 \delta+8 \varepsilon+4 r)} \cdot\left(1-\frac{3 \varepsilon}{2 \alpha}\right)^{2} \cdot \frac{\left(\int_{0}^{\infty}(\sinh t)^{n} e^{-c\left(1-\frac{3 \varepsilon}{\varepsilon_{0}}\right) t} d t\right)^{2}}{\left(\int_{0}^{\infty}(\sinh t)^{n} e^{-c\left(1-\frac{3 \varepsilon}{2 \alpha}\right)^{-1} t} d t\right)^{2}} \cdot\left(1+c_{1} k^{2} r^{2}\right)^{2} \cdot n
\end{gathered}
$$

Let $c=\frac{n\left(1+\varepsilon^{\prime}\right)}{1-\frac{3 \varepsilon}{\varepsilon_{0}}}$, using the computations made in the proof of the Lemma 2.8, and in particular formula (2.15), we get that:

$$
\begin{gathered}
\left(\frac{\int_{0}^{\infty}(\sinh t)^{n} e^{-c\left(1-\frac{3 \varepsilon}{\varepsilon_{0}}\right) t} d t}{\int_{0}^{\infty}(\sinh t)^{n} e^{-c\left(1-\frac{3 \varepsilon}{2 \alpha}\right)^{-1} t} d t}\right)=\frac{J_{n}\left[c\left(1-\frac{3 \varepsilon}{\varepsilon_{0}}\right)\right]}{J_{n}\left[c\left(1-\frac{3 \varepsilon}{2 \alpha}\right)^{-1}\right]}= \\
\left(1-\frac{3 \varepsilon}{\varepsilon_{0}}\right)^{-(n+1)}\left(1-\frac{3 \varepsilon}{2 \alpha}\right)^{-(n+1)} \cdot\left[\frac{\left(1+\varepsilon^{\prime}\right)-\left(1-\frac{3 \varepsilon}{\varepsilon_{0}}\right)\left(1-\frac{3 \varepsilon}{2 \alpha}\right)}{\varepsilon^{\prime}}\right] . \\
\cdot \prod_{i=0}^{n-1}\left[\frac{n\left(1+\varepsilon^{\prime}\right)+(n-2 i)\left(1-\frac{3 \varepsilon}{\varepsilon_{0}}\right)\left(1-\frac{3 \varepsilon}{2 \alpha}\right)}{2 n+n \varepsilon^{\prime}-2 i}\right] \\
\leq\left(1-\frac{3 \varepsilon}{\varepsilon_{0}}\right)^{-(n+1)}\left(1-\frac{3 \varepsilon}{2 \alpha}\right)^{-(n+1)}\left(1+\frac{8 \varepsilon}{\varepsilon^{\prime} \varepsilon_{0}}\right)\left(1+4(n-2) \frac{\varepsilon}{\varepsilon_{0}}\right)^{n} .
\end{gathered}
$$


Vol. LVI (2018) Volumes Comparison in the presence of a Gromov-Hausdorff ...125

We thus get, making $r<\frac{\varepsilon}{4}$ :

$$
\begin{gathered}
\sum_{i=1}^{n}\left\|d_{\bar{y}} \bar{H}_{r}\left(\mathbf{e}_{i}^{\prime}\right)\right\|^{2} \leq n e^{c(5 \delta+9 \varepsilon)}\left(1-\frac{3 \varepsilon}{\varepsilon_{0}}\right)^{-2(n+1)}\left(1-\frac{3 \varepsilon}{2 \alpha}\right)^{-2 n} . \\
\cdot\left(1+\frac{8 \varepsilon}{\varepsilon^{\prime} \varepsilon_{0}}\right)^{2}\left(1+4(n-2) \frac{\varepsilon}{\varepsilon_{0}}\right)^{2 n} \leq \\
\leq n(1+3 c(5 \delta+9 \varepsilon))\left(1+12(n-1) \frac{\varepsilon}{\varepsilon_{0}}\right)\left(1+18 n \frac{\varepsilon}{\varepsilon_{0}}\right) . \\
\cdot\left(1+16 n(n-2) \frac{\varepsilon}{\varepsilon_{0}}\right)\left(1+9 \frac{n}{c-n} \frac{\varepsilon}{\varepsilon_{0}}\right)
\end{gathered}
$$

where the last inequality deduces from inequalities (a) and (b) of the proof of the Lemma 2.8.

Let us fix $c+1$, if $\varepsilon<\frac{1}{64 n^{2}} \min \left(\varepsilon_{0}, 1\right)$ we may apply the Lemma 2.8 and thus get

$$
\delta^{2} \leq 16 n^{2} \frac{\varepsilon}{\varepsilon_{0}}+18 n \frac{\varepsilon}{\varepsilon_{0}}+12(n+1) \varepsilon
$$

and thus $\delta \leq 5 n \sqrt{\frac{\varepsilon}{\varepsilon_{0}}}+3 n \sqrt{\varepsilon}$. Replacing in the above inequality and using the inequality (c) of the proof of the Lemma 2.8, we obtain:

$$
\begin{gathered}
\sum_{i=1}^{n}\left\|d_{\bar{y}} \bar{H}_{r}\left(\mathbf{e}_{i}^{\prime}\right)\right\|^{2} \leq n\left(1+75 n(n+1) \sqrt{\frac{\varepsilon}{\varepsilon_{0}}}+48 n(n+1) \sqrt{\varepsilon}\right) . \\
\left(1+32 n(n+1) \frac{\varepsilon}{\varepsilon_{0}}\right) \leq n\left(1+160 n(n+1) \sqrt{\frac{\varepsilon}{\min \left(\varepsilon_{0}, 1\right)}}\right) .
\end{gathered}
$$

We conclude by noticing that, for any endomorphism $A$ of an Euclidean space and for any orthonormal basis $\left\{\mathbf{e}_{i}\right\}$ of this space one has

$$
|\operatorname{det} A| \leq\left(\frac{1}{n} \sum_{i=1}^{n}\left\|A\left(\mathbf{e}_{i}\right)\right\|^{2}\right)^{\frac{n}{2}}
$$

and thus deduce from the above inequality that

$$
\left|\operatorname{Jac} \bar{H}_{r}\right| \leq\left(1+160 n(n+1) \sqrt{\frac{\varepsilon}{\min \left(\varepsilon_{0}, 1\right)}}\right)^{\frac{n}{2}} .
$$




\section{The volumes comparison}

A direct consequence of the estimation (3.10) is the comparison of the volumes of the manifolds $(M, g)$ and $\left(X, g_{0}\right)$, however this problem (and its solution given by the following theorems and corollaries) requires some extra assumption. We send to [7] to present some example and counterexample showing the necessity of all these extra assumptions.

Let us now suppose that $h$ is a continuous Hausdorff $\varepsilon$-approximation, then its degree is correctly defined. By the Lemma 2.5, the map $\bar{H}_{r}$ is equivariant with respect to the two actions of $\Lambda$ on $\mathbb{H}^{n}$ and on $\bar{M}$, thus it induces a quotient-map $H_{r}: M \rightarrow X$ which is $C^{1}$ (by the Lemma 3.2) and also has a correctly defined degree. We then have the following:

Lemma 4.1. When $h$ is continuous, the maps $h$ and $H_{r}: M \rightarrow X$ are homotopic, thus they have the same degree.

Proof. Let us consider the probability measure $\mu_{\bar{y}}$ on $\mathbb{H}^{n}$, defined by

$$
\mu_{\bar{y}}=\left(\frac{e^{-c \varrho_{r}(\bar{y}, \tilde{f}(\bullet))}}{\int_{\mathbb{H}^{n}} e^{-c \varrho_{r}(\bar{y}, \tilde{f}(\tilde{z}))} d v_{\tilde{g}_{0}}(\tilde{z})}\right) d v_{\tilde{g}_{0}} .
$$

If $\delta_{\tilde{x}}$ denotes the Dirac measure at the point $\tilde{x}$, from the fact that

$$
\tilde{x}^{\prime} \mapsto \int_{\mathbb{H}^{r}} \cosh \left(\varrho_{\mathbb{H}^{n}}\left(\tilde{x}^{\prime}, \tilde{z}\right)\right) d\left(\delta_{\tilde{x}}\right)(\tilde{z})=\cosh \left(\varrho_{\mathbb{H}^{n}}\left(\tilde{x}^{\prime}, \tilde{x}\right)\right)
$$

attains its minimum when $\tilde{x}^{\prime}=\tilde{x}$, and from the Lemma 2.2 we deduce that $\tilde{x}=\operatorname{bar}\left(\delta_{\tilde{x}}\right)$ and that $\tilde{h}(\bar{y})=\operatorname{bar}\left(\delta_{\tilde{h}(\bar{y})}\right)$.

In the affine space of probability measures on $\mathbb{H}^{n}$, let us consider the path $t \mapsto \nu \frac{t}{\bar{y}}$, defined by $\nu_{\bar{y}}^{t}=(1-t) \mu_{\bar{y}}+t \delta_{\tilde{h}(\bar{y})}$. For every $t \in[0,1]$, the measure $\nu \frac{t}{y}$ satisfies the equivariance property: in fact

$$
\begin{aligned}
& \nu_{\gamma \bar{y}}^{t}=(1-t) \mu_{\gamma \bar{y}}+t \delta_{\tilde{h}(\gamma \bar{y})}=(1-t) \mu_{\gamma \bar{y}}+t \delta_{\bar{r}^{-1}(\gamma) \tilde{h}(\bar{y})}= \\
& \quad=(1-t)\left(\bar{r}^{-1}(\gamma)\right)_{*} \mu_{\bar{y}}+t\left(\bar{r}^{-1}(\gamma)\right)_{*} \delta_{\tilde{h}(\bar{y})}=\left(\bar{r}^{-1}(\gamma)\right)_{*} \nu_{\bar{y}}^{t} .
\end{aligned}
$$

- Let us define $\bar{\Psi}_{t}(\bar{y})=\operatorname{bar}\left(\nu \frac{t}{y}\right)$. As $\operatorname{bar}\left(\gamma_{*} \mu\right)=\gamma(\operatorname{bar}(\mu))$ (see Lemma 2.5) for any isometry $\gamma$ and for any measure $\mu$ satisfying the assumption of the definition of the barycentre, we get:

$$
\begin{aligned}
& \bar{\Psi}_{t}(\gamma \cdot \bar{y})=\operatorname{bar}\left(\nu_{\gamma \bar{y}}^{t}\right)=\operatorname{bar}\left[\left(\bar{r}^{-1}(\gamma)\right)\right]_{*} \nu_{\bar{y}}^{t} \\
& =\left(\bar{r}^{-1}(\gamma)\right) \operatorname{bar}\left(\nu_{\bar{y}}^{t}\right)=\left(\bar{r}^{-1}(\gamma)\right) \circ \bar{\Psi}_{t}(\bar{y}),
\end{aligned}
$$

and thus the map $\bar{\Psi}_{t}$ provides (by quotient) a map $\Psi_{t}: M \rightarrow \mathbb{H}^{n} / \Lambda$. 
Vol. LVI (2018) Volumes Comparison in the presence of a Gromov-Hausdorff ...127

- As $\nu_{\bar{y}}^{0}=\mu_{\bar{y}}$ and $\nu_{\bar{y}}^{1}=\delta_{\tilde{h}(\bar{y})}$, we have $\bar{\Psi}_{0}=\bar{H}_{r}$ and $\bar{\Psi}_{1}=\tilde{h}$ and thus $\Psi_{0}=H_{r}$ and $\Psi_{1}=h$.

- $(t, \bar{y}) \mapsto \bar{\Psi}_{t}(\bar{y})$ is a continuous map:

Proof. For the sake of simplicity, in the sequel, we denote by $d v_{\tilde{g}_{0}}(\tilde{z})$ by $d \tilde{z}$. As $h$ is $C^{0}$, it is a limit (in the $C^{0}$-topology) of a sequence $h_{k}$ of $C^{\infty}$ maps $M^{n} \rightarrow X$ and, for $k$ great enough, $h_{k}$ is homotopic to $h$. As $h_{k}$ lifts to a $C^{\infty}$-equivariant map $\tilde{h}_{k}: \bar{M} \rightarrow \mathbb{H}^{n}$, we define $\nu_{\bar{y}, k}^{t}$ to be $(1-t) \mu_{\bar{y}}+t \delta_{\tilde{h}_{k}(\bar{y})}$ and $\bar{\Psi}_{t}^{k}(\bar{y})=\operatorname{bar}\left(\nu_{\bar{y}, k}^{t}\right)$.

Let $\Phi:[0,1] \times \mathbb{H}^{n} \times \bar{M} \rightarrow \mathbb{R}^{n}$ be defined by $\Phi=\left(\phi_{1}, \ldots, \phi_{n}\right)$, where $\phi_{i}$ is defined by:

$$
\phi_{i}(t, \tilde{x}, \bar{y})=\left(\int_{\mathbb{H}^{n}} e^{-c \varrho_{r}(\bar{y}, \tilde{f}(\tilde{z}))} d \tilde{z}\right)\left(\left.\int_{\mathbb{H}^{n}} d[\cosh \tilde{\varrho}]\right|_{(\tilde{x}, \tilde{z})}\left(\frac{\partial}{\partial \tilde{x}_{i}}\right) d \nu_{\bar{y}, k}^{t}(\tilde{z})\right)
$$

where $m \rightarrow\left(\tilde{x}_{1}(m), \ldots, \tilde{x}_{n}(m)\right)$ is a (globally defined) coordinate system $\mathbb{H}^{n} \rightarrow \mathbb{R}^{n}$. From the fact that $\bar{\Psi}_{t}^{k}(\bar{y})=\operatorname{bar}\left(\nu_{\bar{y}, k}^{t}\right)$, we deduce that $\bar{\Psi}_{t}^{k}(\bar{y})$ is the (unique) solution $\tilde{x}$ of the equation $\Phi(t, \tilde{x}, \bar{y})=\mathbf{0}$.

Let $\mathbf{A}=\left(A_{1}, \ldots, A_{n}\right), \mathbf{B}=\left(B_{1}, \ldots, B_{n}\right), C$ and $\mathbf{D}=\left(D_{1}, \ldots, D_{n}\right)$ be the $\mathbb{R}^{n}$-valued functions defined by:

$$
\begin{gathered}
A_{i}(\tilde{x}, \bar{y})=\left.\int_{\mathbb{H}^{n}} d[\cosh \tilde{\varrho}]\right|_{(\tilde{x}, \tilde{z})}\left(\frac{\partial}{\partial \tilde{x}_{i}}\right) e^{-c \varrho_{r}(\bar{y}, \tilde{f}(\tilde{z}))} d \tilde{z} \\
B_{i}(\tilde{x}, \bar{y})=C(\bar{y}) \cdot D_{i}(\tilde{x}, \bar{y})
\end{gathered}
$$

where

$$
\begin{gathered}
C(\bar{y})=\int_{\mathbb{H}^{n}} e^{-c \varrho_{r}(\bar{y}, \tilde{f}(\tilde{z}))} d \tilde{z} \\
D_{i}(\tilde{x}, \bar{y})=\left.\int_{\mathbb{H}^{n}} d[\cosh \tilde{\varrho}]\right|_{(\tilde{x}, \tilde{z})}\left(\frac{\partial}{\partial \tilde{x}_{i}}\right) d\left(\delta_{\tilde{h}_{k}(\bar{y})}\right)(\tilde{z})
\end{gathered}
$$

by continuity, we have decided that

$$
\left.d[\cosh \tilde{\varrho}]\right|_{(\tilde{x}, \tilde{x})}\left(\frac{\partial}{\partial \tilde{x}_{i}}\right)=\left.\lim _{\tilde{z} \rightarrow \tilde{x}} d[\cosh \tilde{\varrho}]\right|_{(\tilde{x}, \tilde{z})}\left(\frac{\partial}{\partial \tilde{x}_{i}}\right) .
$$

From the definition of $\Phi$ and $\nu_{\bar{y}, k}^{t}$, the equation (4.1) writes:

$$
\Phi(t, \tilde{x}, \bar{y})=(1-t) \mathbf{A}(\tilde{x}, \bar{y})+t \mathbf{B}(\tilde{x}, \bar{y}) .
$$

When $c>\frac{n}{a-\frac{3 \varepsilon}{\varepsilon_{0}}}$, the same arguments as in the proof of the Lemma 2.4, implies that, for every $i, A_{i}, C$ and $D_{i}$ are well defined, because the corresponding integrands are integrable. 
- Since $\Phi$ is derivable with respect to $t$, it follows that

$$
\frac{\partial \Phi}{\partial t}=-\mathbf{A}(\tilde{x}, \bar{y})+\mathbf{B}(\tilde{x}, \bar{y})
$$

which is independent on $t$, and thus continuous with respect to $(t, \tilde{x}, \bar{y})$ if and only if it is continuous with respect to $(\tilde{x}, \bar{y})$; moreover, for all $\tilde{x} \in B_{\mathbb{H}^{n}}\left(\tilde{x}_{0}, 1\right)$ and for all $\bar{y} \in B_{\bar{M}}\left(\bar{y}_{0}, 1\right)$ the properties of the regularized distance function and the triangle inequality allow to write:

$$
\left\|\nabla(\cosh \varrho)_{(\tilde{x}, \tilde{z})}\right\| e^{-c \varrho_{r}(\bar{y}, \tilde{f}(\tilde{z}))} \leq \cosh \left[\tilde{\varrho}_{\left(\tilde{x}_{0}, \tilde{z}\right)}+1\right] e^{-c\left[\varrho_{\bar{M}}\left(\bar{y}_{0}, \tilde{f}(\tilde{z})\right)-r-1\right]} .
$$

As the right-hand side of 4.2 is integrable on $\mathbb{H}^{n}$ with respect to $d v_{\tilde{g}_{0}}$ and is independent on $(\tilde{x}, \bar{y})$, the Lebesgue Dominate Convergence Theorem allows to deduce the continuity, with respect to $(\tilde{x}, \bar{y})$, of the function $\mathbf{A}(\tilde{x}, \bar{y})$ from the continuity of the integrand function. In the same way, we prove that $C$ is a continuous function. Moreover, the map $D_{i}$ :

$$
\begin{aligned}
& (\tilde{x}, \bar{y}) \mapsto \int_{\mathbb{H}^{n}} d[\cosh \tilde{\varrho}]_{(\tilde{x}, \tilde{z})}\left(\frac{\partial}{\partial \tilde{x}_{i}}\right) d\left(\delta_{\tilde{h}_{k}(\bar{y})}\right)(\tilde{z})= \\
= & d[\cosh \tilde{\varrho}]_{\left(\tilde{x}, \tilde{h}_{k}(\bar{y})\right)}\left(\frac{\partial}{\partial \tilde{x}_{i}}\right) \quad\left(=0, \quad \text { if } \quad \tilde{x}=\tilde{h}_{k}(\bar{y})\right)
\end{aligned}
$$

is continuous with respect to $(\tilde{x}, \bar{y})$ since $\tilde{h}_{k}$ is continuous and the $\operatorname{map}(\tilde{x}, \tilde{u}) \mapsto \cosh \tilde{\varrho}_{(\tilde{x}, \tilde{u})}$ is $C^{\infty}$. We then conclude that $\mathbf{B}(\tilde{x}, \bar{y})$ is a continuous function of the couple $(\tilde{x}, \bar{y})$, that $\frac{\partial \Phi}{\partial t}$ is a continuous function of the couple $(\tilde{x}, \bar{y})$ and that $\frac{\partial \Phi}{\partial t}$ is a continuous function of the triple $(t, \tilde{x}, \bar{y})$.

- By definition, we have:

$$
D_{i}(\tilde{x}, \bar{y})=\left.d[\cosh \tilde{\varrho}]\right|_{\left(\tilde{x}, \tilde{h}_{k}(\bar{y})\right)}\left(\frac{\partial}{\partial \tilde{x}_{i}}\right)
$$

which is a continuously differentiable function because $\cosh (\tilde{\varrho})(\tilde{x}, \tilde{z})$ is a $C^{\infty}$ with respect to $(\tilde{x}, \tilde{z})$ (this is easily verified outside the diagonal, on a neighbourhood of the diagonal, this deduces from the fact that $\cosh (\tilde{\varrho})(\tilde{x}, \tilde{z})$ may be written as a $C^{\infty}$ function of $\left.\tilde{\varrho}^{2}(\tilde{x}, \tilde{z})\right)$

Let $a_{i}(\tilde{x}, \bar{y}, \tilde{z})=\left.d[\cosh \tilde{\varrho}]\right|_{(\tilde{x}, \tilde{z})}\left(\frac{\partial}{\partial \tilde{x}_{i}}\right) e^{-c \varrho_{r}(\bar{y}, \tilde{f}(\tilde{z}))}\left(a_{1}\right.$ is the integrand of the function $A_{i}$ ). 
Vol. LVI (2018) Volumes Comparison in the presence of a Gromov-Hausdorff ...129

Then, by definition of covariant derivative,

$$
\begin{gathered}
\frac{\partial a_{i}}{\partial \tilde{x}_{j}}(\tilde{x}, \bar{y}, \tilde{z})=\left.D d[\cosh \tilde{\varrho}]\right|_{(\tilde{x}, \tilde{z})}\left(\frac{\partial}{\partial \tilde{x}_{j}} \frac{\partial}{\partial \tilde{x}_{i}}\right) e^{-c \varrho_{r}(\bar{y}, \tilde{f}(\tilde{z}))}+ \\
+\left.d[\cosh \tilde{\varrho}]\right|_{(\tilde{x}, \tilde{z})}\left(D_{\frac{\partial}{\partial \tilde{x}_{j}}} \frac{\partial}{\partial \tilde{x}_{i}}\right) e^{-c \varrho_{r}(\bar{y}, \tilde{f}(\tilde{z}))}= \\
=\cosh [\tilde{\varrho}(\tilde{x}, \tilde{z})] \cdot \tilde{g}_{0}\left(\frac{\partial}{\partial \tilde{x}_{j}}, \frac{\partial}{\partial \tilde{x}_{i}}\right) e^{-c \varrho_{r}(\bar{y}, \tilde{f}(\tilde{z}))}+ \\
\left.\quad \sinh [\tilde{\varrho}(\tilde{x}, \tilde{z})] \cdot d \tilde{\varrho}\right|_{(\tilde{x}, \tilde{z})}\left(D_{\frac{\partial}{\partial \tilde{x}_{j}}} \frac{\partial}{\partial \tilde{x}_{i}}\right) e^{-c \varrho_{r}(\tilde{y}, \tilde{f}(\tilde{z}))}
\end{gathered}
$$

From this, and from the fact that, the coordinate system being fixed, $\left\|\frac{\partial}{\partial \tilde{x}_{i}}\right\|$ and $\left\|D_{\frac{\partial}{\partial \tilde{x}_{j}}} \frac{\partial}{\partial \tilde{x}_{i}}\right\|$ are bounded in every compact set, and in particular on $B_{\mathbb{H}^{n}}\left(\tilde{x}_{0}, 1\right)$, we deduce that, for every $\tilde{x} \in$ $B_{\mathbb{H}^{n}}\left(\tilde{x}_{0}, 1\right)$ and $\bar{y} \in B_{\bar{M}}\left(\bar{y}_{0}, 1\right)$

$$
\begin{gathered}
\left\|\frac{\partial a_{i}}{\partial \tilde{x}_{j}}(\tilde{x}, \bar{y}, \tilde{z})\right\| \leq C_{1} \cdot \cosh [\varrho(\tilde{x}, \tilde{z})] e^{-c \varrho_{r}(\bar{y}, \tilde{f}(\tilde{z}))} \leq \\
\leq C_{1} \cdot \cosh \left[\tilde{\varrho}\left(\tilde{x}_{0}, \tilde{z}\right)+1\right] e^{-c\left(\varrho_{r}\left(\bar{y}_{0}, \tilde{f}(\tilde{z})\right)+1+r\right)} .
\end{gathered}
$$

As the right-hand side of this inequality does not depend on $(\tilde{x}, \bar{y})$ and is integrable with respect to $\tilde{z}$ (under the usual assumption $\left.c>\frac{n}{1-\frac{3 \varepsilon}{\varepsilon_{0}}}\right)$ and as the integrand $\frac{\partial a_{i}}{\partial \tilde{x}_{j}}$ depends continuously on $(\tilde{x}, \bar{y})$, the bounded derivability/continuity theorem of Lebesgue implies that $A_{i}$ is continuously differentiable to respect $\tilde{x}$ and that

$$
\begin{gathered}
\frac{\partial A_{i}}{\partial \tilde{x}_{j}}(\tilde{x}, \bar{y})=\int_{\mathbb{H}^{n}} \frac{\partial a_{i}}{\partial \tilde{x}_{j}}(\tilde{x}, \bar{y}, \tilde{z}) d \tilde{z}= \\
=\tilde{g}_{0}\left(\frac{\partial}{\partial \tilde{x}_{j}}, \frac{\partial}{\partial \tilde{x}_{i}}\right) \int_{\mathbb{H}^{n}} \cosh [\tilde{\varrho}(\tilde{x}, \tilde{z})] \cdot e^{-\varrho_{r}(\bar{y}, \tilde{f}(\tilde{z}))} d \tilde{z}+ \\
+\left.\int_{\mathbb{H}^{n}} d[\cosh \tilde{\varrho}]\right|_{(\tilde{z}, \tilde{z})}\left(D_{\frac{\partial}{\partial \tilde{x}_{j}}} \frac{\partial}{\partial \tilde{x}_{i}}\right) \cdot e^{-c \varrho_{r}(\bar{y}, \tilde{f}(\tilde{z}))} d \tilde{z} .
\end{gathered}
$$

Similarly, let us fix a local coordinate system $m \mapsto\left(\bar{y}_{1}(m), \ldots, \bar{y}_{n}(m)\right)$ of $\bar{M}$, which is defined in some geodesic ball $B_{\bar{M}}\left(\bar{y}_{0}, 2 r_{1}\right)$ centred at some arbitrary fixed point $\bar{y}_{0} \in \bar{M}$, the $\frac{\partial}{\partial \bar{y}_{j}}$ 's are then bounded on $B_{\bar{M}}\left(\bar{y}_{0}, r_{1}\right)$. As $\varrho_{r}$ is regular, $a_{i}(\tilde{x}, \bar{y}, \tilde{z})$ is derivable with respect to 
$\bar{y}_{j}$ and these derivatives are continuous with respect $(\tilde{x}, \bar{y})$, moreover it writes:

$$
\begin{gathered}
\frac{\partial a_{i}}{\partial \bar{y}_{j}}(\tilde{x}, \bar{y}, \tilde{z})=-c d(\cosh \varrho)_{(\tilde{x}, \tilde{z})}\left(\frac{\partial}{\partial \tilde{x}_{i}}\right) . \\
\cdot \bar{g}\left(\nabla \varrho_{r}(\bar{y}, \tilde{f}(\tilde{z})), \frac{\partial}{\partial \bar{y}_{j}}\right) e^{-c \varrho_{r}(\bar{y}, \tilde{f}(\tilde{z}))} d \tilde{z}
\end{gathered}
$$

noticing that $\|\nabla \tilde{\varrho}\|$ and $\left\|\varrho_{r}\right\|$ are bounded everywhere, we get

$$
\begin{gathered}
\left|\frac{\partial a_{i}}{\partial \bar{y}_{j}}(\tilde{x}, \bar{y}, \tilde{z})\right| \leq C_{2} \sinh [\tilde{\varrho}(\tilde{x}, \tilde{z})] e^{-c \varrho_{r}(\bar{y}, \tilde{f}(\tilde{z}))} d \tilde{z} \leq \\
\leq C_{2} \sinh \left[\tilde{\varrho}\left(\tilde{x}_{0}, \tilde{z}\right)+1\right] e^{-c\left(\varrho_{r}\left(\bar{y}_{0}, \tilde{f}(\tilde{z})\right)+r_{1}+r\right)} d \tilde{z}
\end{gathered}
$$

for every $(\tilde{x}, \bar{y}, \tilde{z})$ such that $\tilde{x} \in B_{\mathbb{H}^{n}}\left(\tilde{x}_{0}, 1\right)$ and $\bar{y} \in B_{\bar{M}}\left(\bar{y}_{0}, r_{1}\right)$. As the right-hand side of this inequality does not depend on $(\tilde{x}, \bar{y})$ and is integrable with respect to $\tilde{z}$ (when $c>\frac{n}{1-\frac{3 \varepsilon}{\varepsilon_{0}}}$ ), the bounded derivability/continuity theorem of Lebesgue proves that $A_{i}$ is continuously differentiable with respect to $\bar{y}_{j}$ 's and that

$$
\frac{\partial A_{i}}{\partial \bar{y}_{j}}(\tilde{x}, \bar{y})=\int_{\mathbb{H}^{n}} \frac{\partial a_{i}}{\partial \bar{y}_{j}}(\tilde{x}, \bar{y}, \tilde{z}) d \tilde{z}
$$

A similar argument proves that $C(\bar{y})$ is continuously differentiable with respect to $\bar{y}$ (and thus, trivially with respect to $\tilde{x}$ ). We thus get that $\mathbf{A}, C$ and $\mathbf{D}$ are $C^{1}$ and thus $\Phi$ is $C^{1}$ because all its partial derivatives are continuous.

Moreover, the equality (4.3) gives:

$$
\begin{gathered}
\frac{\partial D_{i}}{\partial \tilde{x}_{j}}(\tilde{x}, \bar{y})=\left.D d[\cosh \tilde{\varrho}]\right|_{\left(\tilde{x}, \tilde{h}_{k}(\bar{y})\right)}\left(\frac{\partial}{\partial \tilde{x}_{i}}, \frac{\partial}{\partial \tilde{x}_{j}}\right) \\
+\left.d[\cosh \tilde{\varrho}]\right|_{\left(\tilde{x}, \tilde{h}_{k}(\bar{y})\right)}\left(D_{\frac{\partial}{\partial \tilde{x}_{j}}} \frac{\partial}{\partial \tilde{x}_{i}}\right) \\
=\tilde{g}_{0}\left(\frac{\partial}{\partial \tilde{x}_{i}}, \frac{\partial}{\partial \tilde{x}_{j}}\right) \cdot \int_{\mathbb{H}^{n}} \cosh \tilde{\varrho}(\tilde{x}, \tilde{z}) d\left(\delta_{\tilde{h}_{k}(\bar{y})}\right)(\tilde{z}) \\
+\left.\int_{\mathbb{H}^{n}} d[\cosh \tilde{\varrho}]\right|_{(\tilde{x}, \tilde{z})}\left(D_{\frac{\partial}{\partial \tilde{x}_{j}}} \frac{\partial}{\partial \tilde{x}_{i}}\right) d\left(\delta_{\tilde{h}_{k}(\bar{y})}\right)(\tilde{z}) .
\end{gathered}
$$


Vol. LVI (2018) Volumes Comparison in the presence of a Gromov-Hausdorff ...131

From this computation and from 4.4, we deduce:

$$
\begin{gathered}
\frac{\partial \Phi_{i}}{\partial \tilde{x}_{j}}=(1-t) \frac{\partial A_{i}}{\partial \tilde{x}_{j}}+t \cdot C(\bar{y}) \cdot \frac{\partial D_{i}}{\partial \tilde{x}_{j}} \\
=\tilde{g}_{0}\left(\frac{\partial}{\partial \tilde{x}_{i}}, \frac{\partial}{\partial \tilde{x}_{j}}\right) \cdot\left[(1-t) \int_{\mathbb{H}^{n}} \cosh \tilde{\varrho}(\tilde{x}, \tilde{z}) e^{-c \varrho_{r}(\bar{y}, \tilde{f}(\tilde{z}))} d \tilde{z}\right. \\
\left.+t \cdot C(\bar{y}) \cdot \int_{\mathbb{H}^{n}} \cosh \tilde{\varrho}(\tilde{x}, \tilde{z}) d\left(\delta_{\tilde{h}_{k}(\bar{y})}\right)(\tilde{z})\right] \\
+\left.(1-t) \int_{\mathbb{H}^{n}} d[\cosh \tilde{\varrho}]\right|_{(\tilde{x}, \tilde{z})}\left(D_{\frac{\partial}{\partial \tilde{x}_{j}}} \frac{\partial}{\partial \tilde{x}_{i}}\right) e^{-c \varrho_{r}(\bar{y}, \tilde{f}(\tilde{z}))} d \tilde{z} \\
+\left.t C(\bar{y}) \int_{\mathbb{H}^{n}} d[\cosh \tilde{\varrho}]\right|_{(\tilde{x}, \tilde{z})}\left(D_{\frac{\partial}{\partial \tilde{x}_{j}}} \frac{\partial}{\partial \tilde{x}_{i}}\right) d\left(\delta_{\tilde{h}_{k}(\bar{y})}\right)(\tilde{z}) \\
=\tilde{g}_{0}\left(\frac{\partial}{\partial \tilde{x}_{i}}, \frac{\partial}{\partial \tilde{x}_{j}}\right) \cdot C(\bar{y}) \int_{\mathbb{H}^{n}} \cosh \tilde{\varrho}(\tilde{x}, \tilde{z}) d \nu_{\bar{y}, k}^{t}(\tilde{z}) \\
+\sum_{l=1}^{n} \Gamma_{j i}^{l} \Phi_{l}(t, \tilde{x}, \bar{y}),
\end{gathered}
$$

the last term being deduced from the definition of the $\Phi_{l}$ 's and from the fact that

$$
D_{\frac{\partial}{\partial \tilde{x}_{j}}} \frac{\partial}{\partial \tilde{x}_{i}}=\sum_{l=1}^{n} \Gamma_{j i}^{l} \frac{\partial}{\partial \tilde{x}_{l}} .
$$

At the point $(t, \tilde{x}, \bar{y})$ such that $\phi(t, \tilde{x}, \bar{y})$, this last term vanishes and we get:

$$
\frac{\partial \Phi_{i}}{\partial \tilde{x}_{j}}=\tilde{g}_{0}\left(\frac{\partial}{\partial \tilde{x}_{i}}, \frac{\partial}{\partial \tilde{x}_{j}}\right) \cdot C(\bar{y}) \int_{\mathbb{H}^{n}} \cosh \tilde{\varrho}(\tilde{x}, \tilde{z}) d \nu_{\bar{y}, k}^{t}(\tilde{z})
$$

as $C(\bar{y})$ and the integral are strictly positive, the matrix $\frac{\partial \phi_{i}}{\partial \tilde{x}_{j}}(t, \tilde{x}, \bar{y})$ is invertible, because the matrix $\tilde{g}_{0}\left(\frac{\partial}{\partial \tilde{x}_{i}}, \frac{\partial}{\partial \tilde{x}_{j}}\right)$ is invertible, being a positive definite symmetric matrix.

The Implicit Function Theorem of U. Dini implies the existence of a $C^{1}$ map $q: V \rightarrow \mathbb{H}^{n}$, where $V \in[0,1] \times \bar{M}$ is an open set containing $\left(t_{0}, \bar{y}_{0}\right)$ such that, in a suitable neighbourhood of $\left(t_{0}, \bar{y}_{0} ; \tilde{x}_{0}=\Psi\left(t_{0}, \bar{y}_{0}\right)\right)$ stands the equivalence:

$$
\Phi(t, \tilde{x}, \bar{y})=0 \Longleftrightarrow \tilde{x}=q(t, \bar{y})
$$


moreover, from the fact that the equivalence

$$
\Phi(t, \tilde{x}, \bar{y})=0 \Longleftrightarrow \tilde{x}=\bar{\Psi}^{k}(t, \bar{y})=\bar{\Psi}_{t}^{k}(\bar{y})
$$

is valid for every $(t, \tilde{x}, \bar{y})$ we deduce the coincidence on $V$ of $\bar{\Psi}_{t}^{k}$ and $q$ : we deduce that $\bar{\Psi}_{t}^{k}$ is $C^{1}$ on a neighbourhood of $\left(t_{0}, \bar{y}_{0}\right)$; as $\left(t_{0}, \bar{y}_{0}\right)$ may be chosen arbitrarily in $[0,1] \times \bar{M}$, we conclude that $\bar{\Psi}^{k}$ is everywhere $C^{1}$ with respect to $(t, \bar{y})$.

- $\bar{\Psi}_{t}^{k}$ is thus a $C^{1}$-homotopy of $\bar{\Psi}_{0}^{k}=\bar{H}_{r}$ on $\bar{\Psi}_{1}^{k}=\tilde{h}_{k}$, moreover each $\bar{\Psi}_{t}^{k}$ is equivariant because, for every $\gamma \in \operatorname{Isom}(\bar{M}, \bar{g})$, one has $\nu_{\gamma \bar{y}, k}^{t}=\left(r^{-1}(\gamma)\right)_{*} \nu_{\bar{y}, k}^{t}$ (because $\mu_{\gamma \bar{y}}=\left(r^{-1}(\gamma)\right)_{*} \mu_{\bar{y}}$ and $\left.\delta_{\tilde{h}_{k}(\gamma \bar{y})}=\delta_{r^{-1}(\gamma) \tilde{h}_{k}(\bar{y})}\right)$ and because for every $l \in I \operatorname{som}\left(\mathbb{H}^{n}, \tilde{g}_{0}\right)$, one has $\operatorname{bar}\left(l_{*} \mu\right)=l[\operatorname{bar}(\mu)]$, see the beginning of the proof of this Lemma, and thus the family $\bar{\Psi}_{t}^{k}$ induces, by quotient, a family of maps $\Psi_{t}^{k}$ which provides a $C^{1}$ homotopy such that $\Psi_{0}^{k}=H_{r}$ and $\Psi_{1}^{k}=h_{k}$. Since $H_{r}$ is homotopic to $h_{k}$, which is homotopic to $h$, we conclude that $H_{r}$ is homotopic to $h$; moreover, since the degree is preserved by homotopy, we conclude that

$$
\operatorname{deg}\left(H_{r}\right)=\operatorname{deg}(h)
$$

The following Theorem gives an estimation of the volumes of the bases $\left(X, g_{0}\right)$ and $(M, g)$ :

Theorem 4.2. Let $\left(X^{n}, g_{0}\right)$ be a compact hyperbolic manifold, let $\varepsilon_{0}$ be a lower bound of the injectivity radius of $\left(X^{n}, g_{0}\right)$. For any oriented manifold $\left(M^{n}, g\right)$, if there exists a continuous Gromov-Hausdorff $\varepsilon$-approximation $h$ : $\left(M^{n}, g\right) \rightarrow\left(X^{n}, g_{0}\right)$ such that $\varepsilon<\frac{1}{64 n^{2}} \min \left(\varepsilon_{0}, 1\right)$, then

$$
\operatorname{Vol}\left(M^{n}, g\right) \geq\left(1+160 n(n+1) \sqrt{\frac{\varepsilon}{\min \left(\varepsilon_{0}, 1\right)}}\right)^{\frac{n}{2}}|\operatorname{deg} h| \cdot \operatorname{Vol}\left(X^{n}, g_{0}\right) .
$$

Proof. Let us denote by $\omega_{g}$ the volume form of $(M, g)$ and $\omega_{g_{0}}$ the one of $\left(\mathbb{H}^{n} / \Lambda, g_{0}\right)$. As $H_{r}$ is regular (by Lemma 3.2 ) we may write

$$
\int_{M} \operatorname{Jac}\left(H_{r}\right) \omega_{g}=\int_{M} H_{r}^{*} \omega_{g_{0}}=\operatorname{deg}\left(H_{r}\right) \int_{X} \omega_{g_{0}}=\operatorname{deg}(h) \int_{X} \omega_{g_{0}}
$$

the last inequality coming from the Lemma 4.1. We deduce that

$$
\int_{M}\left|\operatorname{Jac}\left(H_{r}\right)\right| d v_{g} \geq|\operatorname{deg}(h)| \operatorname{Vol}\left(\mathbb{H}^{n} / \Lambda, g_{0}\right) .
$$


Vol. LVI (2018) Volumes Comparison in the presence of a Gromov-Hausdorff ...133

As $\left|\operatorname{Jac}\left(H_{r}\right)\right|$ is everywhere bounded (from above) by

$$
\begin{aligned}
& \left(1+160 n(n+1) \sqrt{\frac{\varepsilon}{\min \left(\varepsilon_{0}, 1\right)}}\right)^{\frac{n}{2}}, \text { the inequality }(4.5) \text { gives } \\
& \quad\left(1+160 n(n+1) \sqrt{\frac{\varepsilon}{\min \left(\varepsilon_{0}, 1\right)}}\right)^{\frac{n}{2}} \operatorname{Vol}(M, g) \geq|\operatorname{deg}(h)| \operatorname{Vol}\left(X, g_{0}\right) .
\end{aligned}
$$

Remark 4.1. In this proof we seem to suppose that the manifold $(M, g)$ is oriented. In fact we do not need to make the orientability assumption: in fact in the above proof, the sign of the Jacobian is not relevant, because the inequality (4.5) uses the absolute value of the Jacobian.

However, in the not-oriented case the above notion of degree is not relevant, we must replace it by the notion of absolute degree (denote by Adeg), with values in $\mathbb{Z} / 2 \mathbb{Z}$, and which only says whether the degree is trivial or not. In this case, the corollary would be:

Corollary 4.3. Under the assumptions of Theorem 4.2, if $M$ is not oriented, when the absolute degree is not trivial, then

$$
\left(1+160 n(n+1) \sqrt{\frac{\varepsilon}{\min \left(\varepsilon_{0}, 1\right)}}\right)^{\frac{n}{2}} \operatorname{Vol}(M, g) \geq \operatorname{Vol}\left(X, g_{0}\right) .
$$

Proof: The co-area formula, (see [3], p. 104-107) gives:

$$
\int_{M}\left|\operatorname{Jac} H_{r}\right|(y) d v_{g}(y)=\int_{\mathbb{H}^{n} / \Lambda} \sharp H_{r}^{-1}(\{x\}) d v_{g_{0}}(x) .
$$

It is thus sufficient to prove that $H_{r}$ is surjective obtaining, for all $x \in \mathbb{H}^{n} / \Lambda$, $\sharp H_{r}^{-1}(\{x\}) \geq 1$. If Adeg $h \neq 0$, by Lemma 4.1 we deduce that $A d e g H_{r} \neq 0$, and thus $H_{r}$ is surjective.

If the $\varepsilon$-approximation $h$ is not continuous, we must notice that the barycentre map $H_{r}$, that we just have constructed, is still regular and its degree is well defined, we thus obtain the more general

Theorem 4.4. Let $\left(X^{n}, g_{0}\right)$ be a compact hyperbolic manifold, let $\varepsilon_{0}$ be a lower bound of the injectivity radius of $\left(X^{n}, g_{0}\right)$. For any oriented manifold $\left(M^{n}, g\right)$, if there exists a measurable Gromov-Hausdorff $\varepsilon$-approximation $h$ : $\left(M^{n}, g\right) \rightarrow\left(X^{n}, g_{0}\right)$ such that $\varepsilon<\frac{1}{64 n^{2}} \min \left(\varepsilon_{0}, 1\right)$, then

$$
\operatorname{Vol}(M, g) \geq\left(1+160 n(n+1) \sqrt{\frac{\varepsilon}{\min \left(\varepsilon_{0}, 1\right)}}\right)^{-\frac{n}{2}}\left|\operatorname{deg} H_{r}\right| \operatorname{Vol}\left(X, g_{0}\right) .
$$


Proof. The proof of this Theorem is the same as the proof of the Theorem 4.2, except for the fact that we no more have to use the equality $\operatorname{deg} H_{r}=\operatorname{deg} h$, i.e. we no more use the Lemma 4.1.

Remark 4.2. The Theorems 4.2 and 4.4 provide a new simpler proof and a generalization of the results that we referred at the beginning of this paper, which also compared the volumes of $(M, g)$ and $\left(X, g_{0}\right)$, i.e. the Theorem 1.1 of G. Besson, G. Courtois and S. Gallot and the Theorem 1.2 of G. Reviron; notice that our result contain no assumption on the geometry (curvature, entropy,...) of $(M, g)$, except the (crude) assumption that there exists a $\varepsilon-$ Gromov-Hausdorff approximation from $(M, g)$ to $\left(X, g_{0}\right)$ (and a no vanishing condition on the degree of $H_{r}$ or $\left.h\right)$. Moreover, in this article we got an extension to the Hyperbolic Manifold what above obtained for the Flat Torus in [7]: both spaces admits an explicit volume form and an easy computation of the barycentre. On the contrary in [8] we obtained analogues estimates for manifold with pinched curvature for which the volume form is not explicit.

\section{References}

[1] G. Courtois G. Besson S. Gallot, Entropies et rigidités des espaces localment symétriques de courbure strictement négative, GAFA, 5, (1995), 731-799

[2] G. Courtois G. Besson S. Gallot, Rigidity of amalgamated products in negative curvature, Journ. of Differerential Geometry, 79, (2008), 335-387

[3] D. Burago V.A. Zallager, Geometric Inequalities, Springer Series in Soviet Mathematics, Springer-Verlag, Berlin, 1988

[4] K. Shiohama K. Grove, A generalized sphere theorem, Annals of Math., 106, (1977), 201-211

[5] G. Reviron, Espaces de longueur d'entropie majorée: rigidité topologique, adhérence des variétés, noyau de la chaleur, Thèse de Doctorat de Mathématiques de l'Université Joseph Fourier, Grenoble, 2005

[6] G. Reviron, Rigidité topologique sous une hypothèse d'entropie majorée et applications, Commentarii Math. Helv., 83, (2008), 815-846

[7] L. Sabatini, Volume Comparison in presence of a Gromov-Hausdorff $\varepsilon-$ approximation I, Math. Z., 274, (2013), 1-20

[8] L. Sabatini, Volume Comparison without curvature assumptions, Math Z., 282, (2016), 691-714 
Vol. LVI (2018) Volumes Comparison in the presence of a Gromov-Hausdorff ...135

Received: 21.07.2017

Accepted: 8.01.2018

Revised: 21.12 .2017 